\title{
How the brain encodes the order of letters in a printed word: The SERIOL model and selective literature review
}

\author{
CAROL WHITNEY \\ University of Maryland School of Medicine, Baltimore, Maryland
}

\begin{abstract}
This paper describes a novel theoretical framework of how the position of a letter within a string is encoded, the SERIOL model (sequential encoding regulated by inputs to oscillations within letter units). Letter order is represented by a temporal activation pattern across letter units, as is consistent with current theories of information coding based on the precise timing of neural spikes. The framework specifies how this pattern is invoked via an activation gradient that interacts with subthreshold oscillations and how it is decoded via contextual units that activate word units. Using mathematical modeling, this theoretical framework is shown to account for the experimental data from a wide variety of string-processing studies, including hemispheric asymmetries, the optimal viewing position, and positional priming effects.
\end{abstract}

\section{INTRODUCTION}

Little is known of how the brain represents information at the higher levels of cognitive processing. We suggest that the problem of how letter position within a word is encoded provides a tractable area of investigationinto this realm. This problem is circumscribed, yet it involves important higher level processes, such as the composition of a representation from constituent entities, and the formation of a representation that is independent of absolute location in visual space. Furthermore, there is a wealth of relevant experimental evidence. So how does the brain represent that the string "ART" is composed of the letters "A," then "R," then "T," and not "R," then "A," then "T," or "T," then "A," then "R"?

Recent computational models of word recognition have used one of two approaches to the coding of letter position. In a channel-specific scheme, it is hypothesized that different sets of letter units exist for each string position. That is, there are separate units representing " $A$ " in the first position, "A" in the second position, and so on (Coltheart, Curtis, Atkins, \& Haller, 1993; McClelland \& Rumelhart, 1981; Whitney, Berndt, \& Reggia, 1996). Encoding the string "ART" corresponds to activating the unit for " $A$ " in the first set, " $R$ " in the second set, and " $T$ " in the third set. A disadvantage of such a scheme is that it demands a high degree of item redundacy, requiring a representation of each letter in each possible position. Moreover, it is not consistent with recent studies indicating

\footnotetext{
The author is grateful to Udaya Shankar, Rita S. Berndt, and James A. Reggia for helpful suggestions. The author also thanks John Wixted, Joseph Hellige, and two anonymous reviewers for their detailed, supportive, and constructive comments. Correspondence should be addressed to C. Whitney, Department of Philosophy, University of Maryland, College Park, MD 20742 (e-mail: cwhitney@cs.umd.edu).
}

that relative order among sets of letters, not their absolute position, is important in letter-position coding (Humphreys, Evett, \& Quinlan, 1990; Peressotti \& Grainger, 1999) and that priming across letter positions can occur (Peressotti \& Grainger, 1995).

In context-unit encoding, the basic unit is not a single letter, but rather a group of ordered letters, usually trigrams (Mozer \& Behrmann, 1992; Seidenberg \& McClelland, 1989). For example, the string "ART" would be represented by activating units representing “_AR," "ART," and "RT_," where "_" represents a word boundary. Such a scheme is more consistent with evidence of the importance of letter order than a channel-specific one. It has been proposed that context units can also be activated by nonadjacent letters (Mozer, 1987). For example, under a nonadjacent scheme, the trigrams "_RT," "_AT," "AT_," and "AR_" would also be activated by the string "ART." Humphreys et al. (1990) have indicated that such a noncontiguous representation is necessary to fully account for experimental results on relative order and have suggested that activations of context units are graded by the proximity of their constituent letters within the string. However, no mechanism has been proposed for generating such activations. Thus, the difficult question of how such contextual units could be recognized and activated remains unaddressed.

Another possible encoding is a letter-tagging scheme, in which an individual letter unit can represent any position, since it is marked for the ordinal position in which it occurred when a word is read. For example, "ART" would be represented by "A-1," "R-2," "T-3." Such a proposal reduces item redundancy and allows for priming across letter positions, since a letter unit can represent any position. However, it is unknown how such tagging could be realized in physiological terms.

In considering the nature of letter-position coding, we believe that results from studies on the perceptibility of letters 
within strings are directly relevant. Such experiments have shown that, for briefly presented letter strings, the first letter has the highest probability of being correctly recognized, the second letter has the second highest probability, with the probability of recognition generally decreasing from left to right (with the possible exceptions of the final letter and the letter at fixation; see Estes, Allmeyer, \& Reder 1976; Lefton, Fisher, \& Kuhn, 1978; Montant, Nazir, \& Poncet, 1998; Wolford \& Hollingsworth, 1974). Initial interpretations of these results postulated a serial scanning process across an input trace (Lefton et al., 1978; Mewhort, Merikle, \& Bryden, 1969). More recently, interpretations have focused instead on weights derived from perceptual factors and/or position of maximal information within a string (Brysbaert, Vitu, \& Schroyens, 1996; Montant et al., 1998; O'Regan, Levy-Schoen, Pynte, \& Brugaillere, 1984).

We suggest another possibility: that this pattern of perceptibility emerges directly from the way in which letter position is encoded. Consistent with earlier interpretations, we suggest that serial processing is involved and that letter position is encoded by a temporal firing pattern across letter units. Specifically, we propose a lettertagging scheme in which the tag corresponds to the time of firing relative to an underlying oscillatory cycle. In keeping with the evidence for the importance of letter order, we suggest that this temporal representation then activates contextual units. Thus, we combine two letter position encoding schemes and address their respective shortcomings, by attempting to specify how letter position is tagged and how context units are activated.

We have dubbed this model the SERIOL framework of letter position encoding (sequential encoding regulated by inputs to oscillations within letter units). Our goal was to construct a theoretical framework for written word recognition that is consistent with psychological studies and to perform computer simulations that reproduce the data from those studies. At the same time, we wanted a framework that is neurobiologically plausible and is consistent with current theories of neural computation and physiology. In pursuit of this goal, we have constructed a model of word recognition that extends from the retinal level to the lexicon and accounts for a wide range of phenomena, from patterns of letter perceptibility across visual fields to hemispheric modes of processing.

The organization of this paper is as follows. First, we present the details of SERIOL framework. Then, we discuss how this framework is consistent with and accounts for data gathered from many studies relevant to various aspects of letter position encoding and word recognition. The results of some of those studies are reproduced via mathematical models based on the proposed framework. Finally, in the Discussion section, we further address the issues introduced here.

\section{THE SERIOL FRAMEWORK}

This section provides a specification of SERIOL model by describing its structure and the functionality of each layer. The SERIOL model provides a theoretical frame- work. The entire framework has not been implemented in a computational model. However, portions have been implemented; those simulations will be described in later sections. We first give an overview of the SERIOL framework, followed by a more detailed description.

The framework is composed of five layers, each having distinct activation dynamics. We will use the term node to refer to the basic computational unit. A node may be thought of as corresponding to a neuronal assembly. Except at the lowest layer, a node's function is to recognize the occurrence of a symbol.

At the lowest processing layer, the retinal level, a node represents a pixel. The nodes in this level are precisely topographically organized with respect to visual input from external space, and perceptual acuity decreases as distance from the visual fixation point increases. At the feature level, nodes tuned to retinal location recognize suborthographic letter features. At this level, the retinal pattern of activation resulting from perceptual factors is transformed to reflect locational information, wherein activation decreases from left to right across location. At the letter level, nodes recognize individual letters. The varying levels of input from the feature level are converted into a temporal firing pattern across the nodes, in which the firing order tags position. This is accomplished by interaction with an underlying subthreshhold oscillation, such that the letter node receiving the most input fires first, the letter node receiving the second highest level of input fires next, and so on. Lateral inhibition ensures that only one letter node fires at a time. At the bigram level, nodes recognize ordered pairs of letters, converting the temporal representation of the letter level to a contextual representation. At the word level, nodes recognize sets of bigrams comprising entire words.

Figures 1 and 2 summarize how the string "CART" would be represented within this framework. Figure 1 diagrams the activation patterns at the retinal and feature levels; Figure 2 displays the letter level to the word level. In summary, at the feature level, an activation gradient is established across the features of the letters; at the letter level, "C" fires, then "A," then "R," then "T"; at the bigram level, "CA," "AR," "RT," "CR," "AT," and "CT" become active; at the word level, "CART" becomes the most active of the word nodes.

We next focus on each processing level in more detail. The higher processing layers of the framework, from the letter to word levels, have been previously described (Whitney \& Berndt, 1999). Therefore, they will not be presented in as much detail as the lower processing levels, which have not previously been specified.

\subsection{Retinal Level}

On the physical retina, visual acuity falls off with increasing angle from the fixation point, due to the decreasing concentration of cones. Thus, in terms of visual acuity, objects at fixation are significantly better perceived than objects outside of fixation; an early study showed that performance in a line detection task decreased more than $50 \%$ within $1^{\circ}$ of the center of the fovea (Alpern, 1962). 


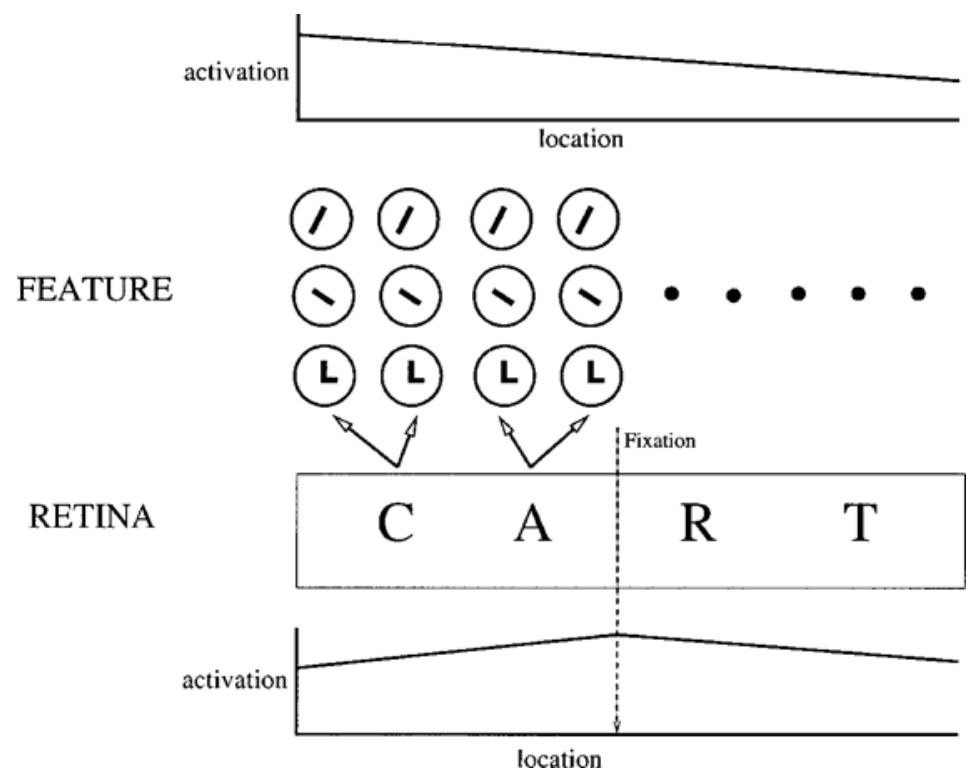

\begin{abstract}
Figure 1. Architecture of the retinal and feature levels of the SERIOL framework. The retinal level is precisely topographically organized with respect to external stimulus-in this case, "CART." The activation of retinal nodes decreases as distance from fixation increases. Feature nodes are tuned to retinal location. The activation of feature nodes decreases from left to right across locations.
\end{abstract}

For clarity of presentation, we will use the term location to refer to physical location in space (with respect to visual fixation) and the term position to refer to a letter's ordinal position within a string. Abstracting away from physical retinas, the retinal level in our framework is fully specified by acuity, $C$, which is a function of retinal location, $R$. For simplicity, we consider location to be onedimensional, specifying horizontal distance from the point of visual fixation (for which $R=0$ ). We take locations to the left of fixation (i.e., in the left visual field, LVF) to have negative values and locations to the right of fixation (in the right visual field, RVF) to have positive values. Acuity is symmetric around fixation; that is, $C(R)=$ $C(-\mathrm{R})$. Acuity is maximal at $R=0$ and decreases with increasing $|R|$. We will denote this pattern of activation (i.e., the function $C$ ) as the acuity gradient. The activation pattern for the retinal layer in Figure 1 corresponds to the acuity gradient.

\subsection{Feature Level}

At the next processing level, nodes recognize suborthographic features. We do not model the process of feature extraction itself, but rather assume that each feature node reaches a level of activation that is consistent with our specifications of bottom-up and lateral patterns of activation. We make the following assumptions. Feature nodes are broadly tuned to retinal location and send directional lateral inhibitory input to each other. Features are independently extracted in each hemisphere. The amount of excitatory and inhibitory input varies with the hemisphere. This hemisphere-specific activation converts the acuity gradient into a locational gradient. We next discuss each of these assumptions in more detail.

It is commonly assumed that letters are identified by analyzing suborthographic features (e.g., lines, angles, and curves). Several studies have indicated that letter features play a role in letter recognition and that similar features occurring in different locations interact with each other, where the degree of interaction depends on the spatial distance between the features (Bjork \& Murray, 1977; Chastain, 1977; Krumhansl \& Thomas, 1976; Strangert \& Brännström, 1975).

As is consistent with the results of these experiments, the next level of our model is composed of feature detectors that are tuned to retinal location. There is neurobiological evidence that the response patterns of neurons can be broadly tuned - that is, a neuron responds most strongly to a specific stimulus, and its response falls off as the similarity to this preferred stimulus decreases. For instance, some neurons in the motor cortex are tuned to movement direction. Such a neuron fires most strongly when movement in space occurs in its preferred direction. As the direction of movement increasingly differs from this preferred direction, the neuron's firing level decreases (Georgopoulos, Kalaska, Caminiti, \& Massey, 1982). We propose a similar pattern of activation for feature nodes with respect to retinal location. That is, a feature node is most highly activated when the feature that it recognizes occurs in a certain optimal area of the retina (the feature node's preferred location), less strongly activated when that feature occurs near the preferred location, and not at all activated when that feature occurs far from the preferred 


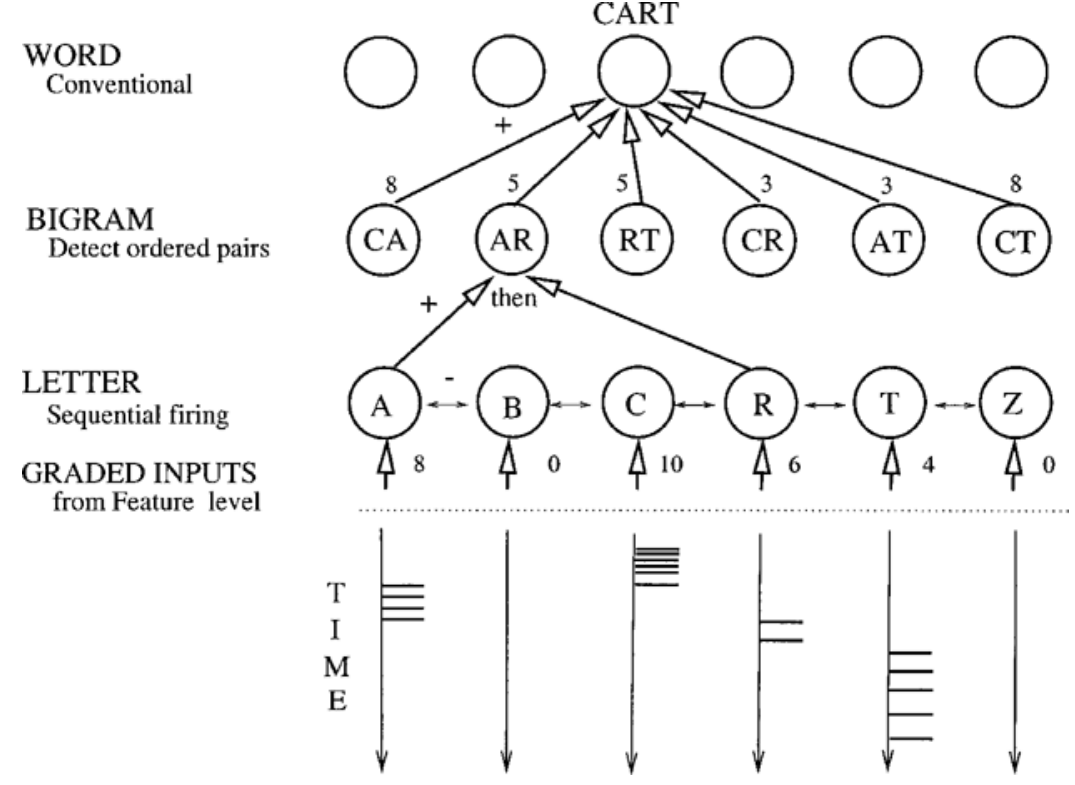

Figure 2. Architecture of the word, bigram, and letter levels of the SERIOL framework, with example of encoding the word "CART." At the letter level, simultaneous graded inputs and lateral inhibition create a temporal firing pattern, as indicated by the timing of firing displayed under the letter nodes. Excitatory connections link the letter nodes and the bigram nodes, which recognize ordered pairs of letters. The activations of the bigrams (shown above the nodes) are proportional to the activations of the constituent letters. Excitatory connections link the bigram nodes and the word nodes. For each word node, connection weights are proportional to the bigram activations evoked by that word. Activation of word nodes is based on conventional neural network models.

location. Each possible feature stimulus is detected by multiple nodes having different preferred locations. This proposal can account for the experimental evidence that similar features interact with each other, where the degree of interaction decreases as the spatial distance between features increases.

The connections from the retinal level to the feature level in our framework correspond to information flow from the retinas to the cerebral hemispheres. The visual system is connected in such a way that information from the LVF is initially processed by the right hemisphere $(\mathrm{RH})$, and information from the RVF is initially processed by the left hemisphere (LH). However, it is not clear whether there is overlap along the vertical meridian between the two visual fields. This issue has important implications for letter string processing. If there is sufficient overlap, all letters within a string are sent directly to both hemispheres. If not, normal processing of letter strings must involve interhemispheric (callosal) transfer in order to integrate the two halves of the string. Brysbaert (1994) reports that many authors assume visual field overlap on the order of $1^{\circ}$ to $3^{\circ}$. However, Brysbaert argues that assumptions for overlap are based on weak, indirect evidence from anatomical findings. On the basis of comparisons of the optimal viewing position for words in subjects presumed to process language in the $\mathrm{RH}$ versus those who process language in the LH, Brysbaert con- cludes that, with respect to reading, functionally there is no overlap and that interhemispheric transfer normally occurs for centrally presented stimuli. This result will be discussed in more detail in Section 3.2. Moreover, experiments with commissurotomy patients and hemianopic patients have provided evidence against such an overlap; if any overlap does exist, it is less than one half of a degree and provides only partial visual information (Fendrich \& Gazzaniga, 1989; Sugishita, Hamilton, Sakuma, \& Hemmi, 1994; Sugishita, Hemmi, Sakuma, Beppu, \& Shiokawa, 1993; Trauzettel-Klosinski \& Reinhard, 1998).

Following this evidence of no visual field overlap, we assume that visual information about letters falling to the left of fixation (the initial letters in English words) is projected to the $\mathrm{RH}$ and that information about letters falling to the right of fixation (the final letters in English words) is projected to the LH. Letter features are extracted separately in each hemisphere.

We propose that the pattern of activation attained by feature nodes is very different from the pattern of activation at the retinal level. Experimentally, it has been noted that the pattern of perception of letters within a string violates the pattern of the acuity gradient. Numerous studies of briefly presented letter strings have shown that the initial letter of a string is the most accurately perceived of all the letters, even when the initial letter is far from fixation (Hammond \& Green, 1982; Lefton et al., 1978; 
Mason, 1982; Wolford \& Hollingsworth, 1974). This pattern cannot be attributed to decreased lateral inhibition for boundary letters; when strings of nonletter symbols are centrally presented, the initial and final symbols are the least well perceived, as would be expected if perception were simply a function of visual acuity (Hammond $\&$ Green, 1982; Mason, 1982). The enhanced perception of the initial symbol also holds for strings of numbers. Thus, it appears that a specialized system exists for analyzing symbols that commonly occur in strings. This system overrides the effects of visual acuity.

In brief, we propose that this string-processing system operates as follows. The acuity gradient of the retinal level is transformed into a locational gradient at the feature level. For languages read from left to right, the locational gradient is characterized by decreasing activation from left to right across preferred location. That is, feature nodes with the leftmost preferred locations attain the highest level of activation, and activation levels decrease for more rightward preferred locations (see the activation pattern for the feature layer in Figure 1). At the letter level, this locational gradient is converted into a temporal firing pattern that encodes position. As a consequence of this encoding process, a positional gradient across letter node activations emerges. This positional gradient underlies experimentally observed patterns of letter recognition within strings.

In the remainder of this section, we specify how the acuity gradient is converted to the locational gradient. For simplicity, we consider only those feature nodes most highly activated by the retinal inputs (i.e., those feature nodes activated by features occurring at their preferred locations). We assume that this conversion mechanism is learned during reading acquisition. We do not address how it is learned, but rather propose a model of information processing for a skilled reader.

Locational gradient formation depends on two important characteristics of the reader: scanning direction and dominant hemisphere for language processing. In the following specification, we assume that words are read from left to right (as in English) by a reader having the usual brain organization-that is, LH dominant. In Section 3, we show how this specification can account for experimentally observed patterns of letter perceptibility, and we consider other combinations of scanning direction and dominant hemisphere.

The proposed locational gradient is monotonically $d e$ creasing from left to right. Note that, for English words, the slope of the acuity gradient in the RVF/LH (from fixation to the final letter) is in the same direction as our proposed locational gradient. In contrast, the slope of the acuity gradient in the LVF/RH (from the initial letter to fixation) is increasing - that is, the slope is in the opposite direction of our proposed locational gradient (for example, compare the activation patterns for the retinal layer and the feature layer in Figure 1). We propose that, in the $\mathrm{LVF} / \mathrm{RH}$, the slope of the acuity gradient is reversed or inverted as it activates letter features, whereas, in the RVF/LH, it is maintained. This occurs via stronger weights in the LVF/RH on bottom-up excitatory connections and on inhibitory connections within the feature level that act from left to right (the differing weight magnitudes across hemispheres and the directional nature of the inhibition were learned during reading acquisition). Following callosal transfer, the resulting two partial gradients are combined, forming a locational gradient that is monotonically decreasing from left to right.

This process of locational gradient formation is illustrated in Figure 3. We assume that letter features in the LVF/RH become more highly activated by retinal inputs than those in the RVF/LH (see the upper right panel of Figure 3). This allows the first letter's features to reach a high level of activation, even if they are far from fixation. This assumption is consistent with experimental results showing that, in the LVF/RH, perceptibility of the initial letter does not decrease as distance from fixation increases, whereas, in the RVF/LH, it does decrease (Estes et al., 1976). (This result will be discussed in greater detail in Section 3.1.) Within the feature level of the RH, we propose that strong directional lateral inhibitory connections exist, such that a feature node inhibits all feature nodes with preferred locations to its right. Thus, the amount of inhibitory input increases as distance from fixation decreases, because more and more features send inhibition from the left. We assume that this directional inhibition is strong enough to override the slope of the acuity gradient, inverting it. Thus, the features comprising the first letter attain a high level of activation (due to strong excitation and lack of lateral inhibition), and activation decreases toward fixation (due to sharply increasing directional lateral inhibition; see the lower left panel of Figure 3.)

In the $\mathrm{RVF} / \mathrm{LH}$, we assume that the weights on both the excitatory and the inhibitory connections are weaker, because the acuity gradient is already in the correct direction, so strong weights are not necessary. Thus, the acuity gradient is essentially maintained at the feature level in the LH (although some directional inhibition may steepen its slope).

Feature information from the RH is then transferred to the LH. We assume that the features from the RH inhibit the activations of the LH features, such that the feature activations of the LH's leftmost letter are lower than those of the RH's rightmost letter. As a result, an activation gradient across all features is created in the LH that is strictly decreasing by left-to-right location (see the lower right panel of Figure 3). Thus, the locational gradient is formed. A mathematical model of this conversion is given in Appendix A.

\subsection{Letter Level}

We propose that the locational gradient of the feature level induces a temporal firing pattern across letter nodes, wherein position is represented by the precise timing of firing relative to other letter nodes. This idea is consistent with current neurobiological models of information encoding. Hopfield (1995) has proposed that quantities are represented by the explicit timing of action potentials, 

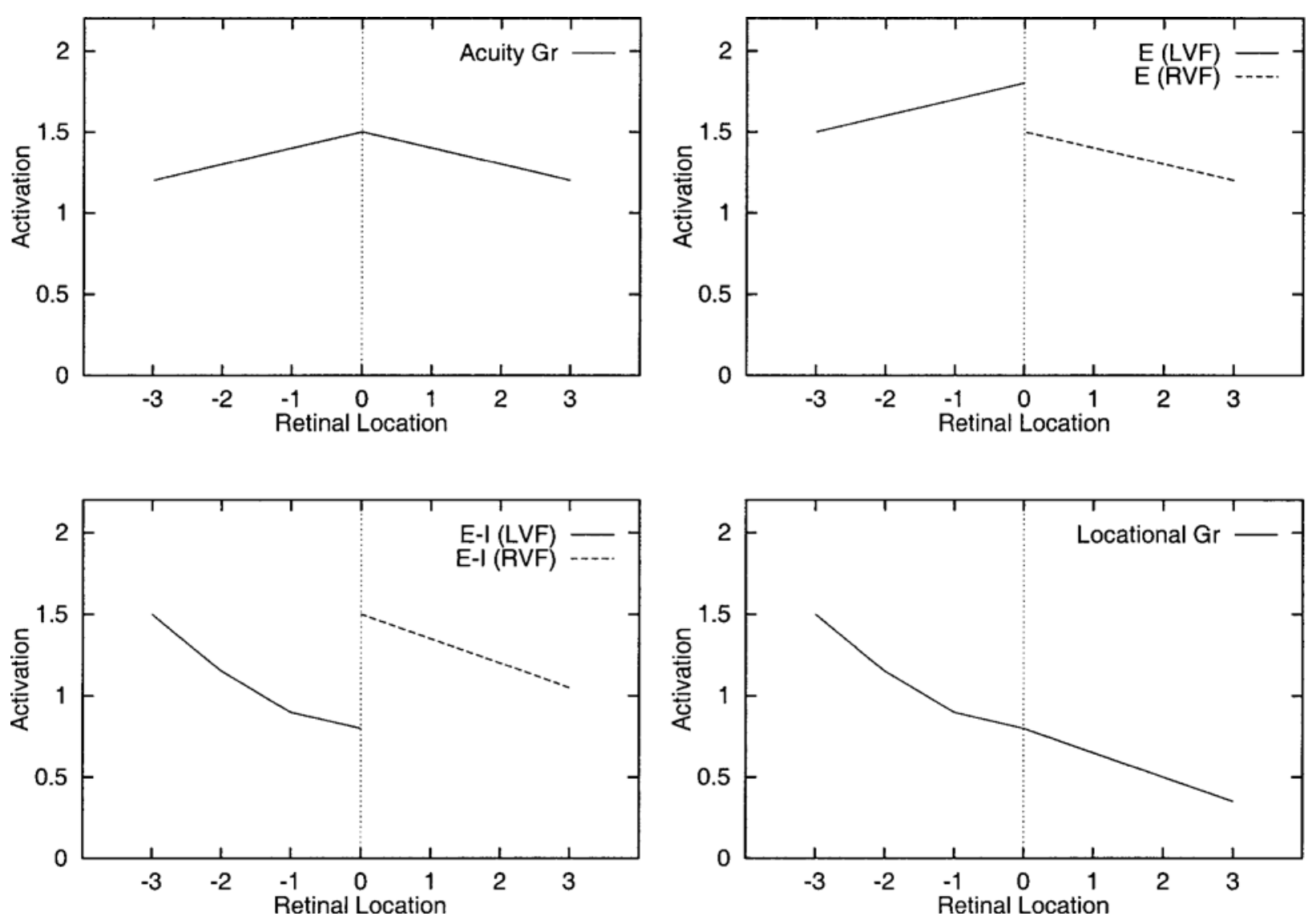

Figure 3. Example formation of the locational gradient across the activations of features. In the upper left graph, the acuity gradient of the retinal level is displayed in abstract units of activation. In the upper right graph, the effects of hemisphere-specific excitation, $E$, at the feature level are shown. Note that in the RVF/LH (retinal location $\geq 0$ ), $E$ is equivalent to the acuity gradient, whereas in the LVF/RH (retinal location $<0$ ), $E$ is elevated with respect to the acuity gradient. In the lower left graph, the effects of hemispherespecific leftward lateral inhibition, $E-I$, are displayed. Note that inhibition is much stronger in the LVF/RH than in the RVF/LH and results in inversion of the direction of the slope of the gradient in the LVF/RH. In the lower right graph, the hemisphere-specific gradients are joined via interhemispheric inhibition of the RVF/LH's features to form a monotonically decreasing activation gradient across feature locations, the locational gradient.

rather than by their firing rate. In this phase-advance model, encoding neurons undergo internal, subthreshhold oscillations of excitability. The magnitude of an input to such a neuron determines when threshhold is exceeded. For a small input, threshhold is not exceeded until late in the cycle when the cell's oscillation brings its potential near threshhold. For a larger input, threshhold is exceeded earlier in the cycle. Thus, the size of an input is represented by spike timing relative to the oscillatory cycle. This scheme implies that individual spikes are much more important than has traditionally been assumed. Indeed, recent studies have shown that single spikes encode signficant amounts of information (Reike, Warland, de Ruyter van Steveninck, \& Bialek 1997) and that spike timing is precise and reproducible at a millisecond time scale (Berry, Warland, \& Meister, 1997; de Ruyter van Steveninck, Lewen, Strong, Koberle, \& Bialek, 1997; Victor \& Purpura, 1996).

It has been proposed that oscillatory activity in the brain near $40 \mathrm{~Hz}$ (gamma frequencies) is related to cog- nitive processing (Tiitinen et al., 1993). There is evidence that individual $40-\mathrm{Hz}$ waves are related to individual auditory stimuli (Joliot, Ribary, \& Llinas, 1994). It has been suggested that short-term memories are encoded on $40-\mathrm{Hz}$ subcycles of a low-frequency (5- to $12-$ $\mathrm{Hz}$ ) oscillation (Lisman \& Idiart, 1995). This proposal is consistent with the observation of nested oscillations recorded in the human cortex in response to auditory stimuli (Llinas \& Ribary, 1993). We suggest that such oscillations also underlie visual language processing. We propose that each letter position corresponds to a successive 25 -msec subcycle within an oscillatory period of $\approx 200 \mathrm{msec}$.

This coding scheme does not employ position-specific letter detectors; all feature nodes are connected to all letter nodes. Any letter node can represent any position, depending on the level of input that it receives and the resulting timing of firing. We do not model the process of letter recognition; we take as given that a mechanism exists to bind the features of a letter together, culminating 
in activation of the correct letter. All letter nodes are assumed to undergo synchronous, periodic oscillations of excitability. Due to the locational gradient, the letter node representing the letter in the first position receives the highest level of excitatory input, the second receives the next highest amount, and so on. The letter node receiving the highest level of input fires first because it reaches threshhold before the others; the letter node receiving the second highest level of input fires next, and so on. Suitable input levels and lateral inhibition ensure that only one letter node fires at a time. We assume that a letter node continues to fire until it receives lateral inhibition and that, once a letter node receives inhibition after it has already fired, it does not fire again in the oscillatory cycle. A precise description of this temporal encoding process is given in Whitney and Berndt (1999).

The level of input affects the activation of the receiving letter node, where higher levels of input cause faster refiring and, therefore, higher levels of activation. Thus, the locational gradient of the feature level creates a positional gradient across the letter nodes. However, the positional gradient does not necessarily have the same shape as the locational gradient, because the positional gradient results from the interaction of the locational gradient with other factors (namely, the internal states of the letter nodes and lateral inhibition between letter nodes). These interactions can result in a pattern of activation at the letter level across positions that is not monotonically decreasing. Specifically, we propose that the final letter continues to fire until the end of the oscillatory cycle, since it is not inhibited by another letter. As a result, the activation of the final letter depends on when in the cycle it starts firing. If it starts firing relatively early in the cycle, it can achieve a higher level of activation than the internal letters. Also, the relative levels of the inputs to adjacent letters can affect activation levels. This can account for the increased perceptibility of a fixated letter. We discuss these phenomena in more detail in Section 4.2 .

\subsection{Bigram and Word Levels}

At the next level of our framework, bigram units are activated by the sequential dynamics of the letter nodes and provide a mechanism for decoding this temporal representation into a nontemporal encoding. Bigram nodes recognize ordered pairs of letters, corresponding to neuronal assemblies that fire only if input " $A$ " is followed by input "B." Such an assembly would not fire if only "A" were received, or if "B" were received prior to "A." A bigram node becomes active if it receives suitable inputs within the time span of an oscillatory cycle; thus, bigram nodes can be activated by noncontiguous letters in the input string. Bigrams were chosen as the contextual units because they are the most basic such units. For simplicity, bigrams connect directly to words, but we do not mean to rule out the possibility that there may be higher levels of organization between bigrams and words.

The activation of a bigram node depends on the activations of the letter nodes representing its constituent let- ters (increasing activation with increasing input levels) and the time separation between the firing of those letter nodes (decreasing activation with increasing separation).

On the basis of their experimental results, Humphreys et al. (1990) have suggested that words are represented by patterns of activations across context units, where activations are scaled by visual factors, such as the proximity of constituent letters and the presence or absence of lateral masking (which depends on letter position). Our specification of bigram unit activation allows a natural formulation of such graded activations.

At the bigram/word interface, the SERIOL framework follows the classical firing-rate model. These connections are modeled using the conventional neural network model methodology, wherein the input to a word node is the sum of the weighted activations from the bigram level. The weight on a connection from a given bigram node to a given word node is proportional to the activation of that bigram when that word is being represented across the bigram nodes. A more detailed specification and simulations are given in Whitney and Berndt (1999).

Our proposed framework of letter position encoding is consistent with a wide range of experimental data. In the following sections, we discuss how our framework can account for the results of many studies, and we use mathematical models to support our proposals. First, we present studies that are relevant to locational gradient formation. Then, we discuss aspects of the letter level, including temporal encoding, the positional gradient, and a group of studies designed to investigate the nature of letter position coding.

\section{STUDIES RELEVANT TO FORMATION OF THE LOCATIONAL GRADIENT}

Our specification of how the locational gradient is formed can account for a variety of experimental results. First, we discuss studies pertaining to perceptibility of individual letters within nonword strings, which reveal visual field differences that we argue arise from the process of locational gradient formation. Then, we present studies concerning optimal viewing position (OVP) and the RVF advantage, and we discuss the interaction of reading direction and dominant hemisphere in those phenomena. Finally, we show how this discussion relates to the proposal of different hemispheric "modes of processing" in reading.

\subsection{Visual Field Effects on Patterns of Perceptibility}

Studies have shown a robust pattern of decreasing letter perceptibility with increasing string position (with the possible exceptions of the fixated letter and the final letter; see Hammond \& Green, 1982; Lefton et al., 1978; Mason, 1982; Wolford \& Hollingsworth, 1974). This overall pattern is generally independent of fixation point. However, string position did interact with visual field in several studies. For example, in Estes et al. (1976), subjects were to identify briefly presented four-letter strings that 
occurred over one of four possible retinal areas: from -8 to -5 , from -5 to -2 , from 2 to 5 , or from 5 to 8 (where units are in letter widths, and fixation is at 0 ). In the LVF/ $\mathrm{RH}$, perceptibility of the initial letter was unaffected by retinal location, with perceptibility at $80 \%$ for locations -8 and -5 . In the RVF/LH, retinal position did affect perceptibility of the initial letter, with perceptibility for location 2 at $80 \%$ and for location 5 at $50 \%$. Thus, in the $\mathrm{LVF} / \mathrm{RH}$, perceptibility of the initial letter did not fall off as acuity decreased (i.e., as distance from fixation increased), as it did in the RVF/LH. Assuming that this difference arises from the feature level, this finding is consistent with the SERIOL framework, wherein features in the $\mathrm{LVF} / \mathrm{RH}$ receive higher levels of excitatory input than those in the RVF/LH, yielding acuity effects on the initial letter in the RVF/LH, but no such effects in the LVF/RH.

In another study, subjects were to report the letters comprising a nine-letter string (Wolford \& Hollingsworth, 1974). In order to deconfound the effects of retinal location and string position, the location of the string was varied with respect to fixation. The location of the string's initial letter varied from 12 letter widths to the left of fixation $(R=-12)$ to 5 letter widths to the right of fixation $(R=5)$. This yielded separate retinal location curves for all string positions and yielded separate string position curves for retinal locations -4 to 4 .

An analysis of the data showed an interaction of string position with visual field. That is, for a given string position and distance from fixation $(|R|)$, the result varied with the direction from fixation. These experimental data are displayed in the upper panels of Figure 4 (we display the results only for the first seven string positions because, for simplicity, we do not consider possible increased activation levels at the end of the string). To summarize, for a given retinal location in the LVF, perceptibility initially drops off quickly with increasing string position and then levels off. However, in the RVF, perceptibility is more smoothly decreasing. For example, at retinal location 3 in the LVF/RH $(R=-3)$, perceptibility drops sharply from $100 \%$ for position 1 to $35 \%$ for position 3 . Perceptibility continues to decrease to $20 \%$ for position 4 but stays roughly constant as position increases from 4 to 7 . In contrast, at the analogous location in the $\mathrm{RVF} / \mathrm{LH}(R=3)$, perceptibility drops from $95 \%$ for position 1 to $55 \%$ for position 3, a smaller drop than in the LVF/RH. Perceptibility drops to $30 \%$ for position 4 and continues to decrease to $5 \%$ for position 7 , rather than stabilizing as in the LVF/RH.

We suggest that this visual field effect arises from the differences in the formation of the locational gradient in the two hemispheres. Recall that, in the RVF/LH, the locational gradient is roughly equivalent to the acuity gradient, whereas in the LVF/RH, formation of the locational gradient requires inversion of the slope of the acuity gradient via strong excitation and inhibition. It follows that the resulting hemisphere-specific locational gradients would not have the same shape as each other. We developed a mathematical model based on this premise that gives a good approximation to this data.
More specifically, we assume that the amount of inhibition received by a letter's features depends on how many letters are to its left, the activation of those letters' features, and the strengths of the inhibitory connections. These assumptions on leftward lateral inhibition underlie the model's ability to recreate the pattern in the experimental data. In the LVF/RH, perceptibility at a given location initially decreases quickly as string position increases, because inhibitory input increases quickly as more letters occur to the left. However, after a certain point, the level of inhibitory input flattens out as more letters occur to the left. This occurs because those additional letters contribute lower and lower levels of inhibitory input because they are farther from fixation, and, thus, their activation levels are lower. As a result, the amount of inhibitory input received increases nonlinearly as string position increases. However, in the RVF/LH, overall levels of lateral inhibition are weaker, so this nonlinear effect is not as strong, resulting in a smoother decrease in perceptibility. The results of the mathematical model are shown in the lower panels of Figure 4. Note that the model accurately recreates the differing patterns of perceptibility by visual field. The details of the model are given in Appendix A.

We suggest that the assumptions underlying the mechanism of locational gradient formation could be tested more directly using the sequential blanking paradigm. When stimuli are presented briefly (for $<20 \mathrm{msec}$ ) across spatial locations, a stimulus that is followed $100 \mathrm{msec}$ later by another stimulus in a neighboring location is not perceived (Mayzner \& Tresselt, 1970). This masking or blanking is sensitive to the form of the stimuli and is thought to arise from lateral inhibition at the level of "geometric analyzers" (i.e., feature detectors). If the intensity of the masked stimuli is sufficiently increased relative to the masking stimuli, then blanking no longer occurs. By testing the minimal increase in intensity required to overcome the blanking for different retinal positions, the locational gradient formation hypothesis could be investigated. It predicts that there should be a directional effect, where masking stimuli on the left exhibit more inhibition than those on the right. It also predicts a visual field effect, where inhibition is greater in the LVF than in the RVF. However, even if such effects were not observed, it would not necessarily imply that the hypothesis is incorrect, because the directional inhibition could occur at higher processing level than that which underlies the sequential blanking effect.

We have seen how proposed processes underlying the formation of a locational gradient can account for experimental results regarding visual field effects in letter identification. Next, we address experiments examining the role of fixation point in the identification of entire words, leading us to generalize the discussion of locational gradient formation to address the effects of reading direction and dominant hemisphere and to consider the processing time required to perform interhemispheric transfer and acuity gradient inversion. 
$\mathrm{LVF} / \mathrm{RH}$
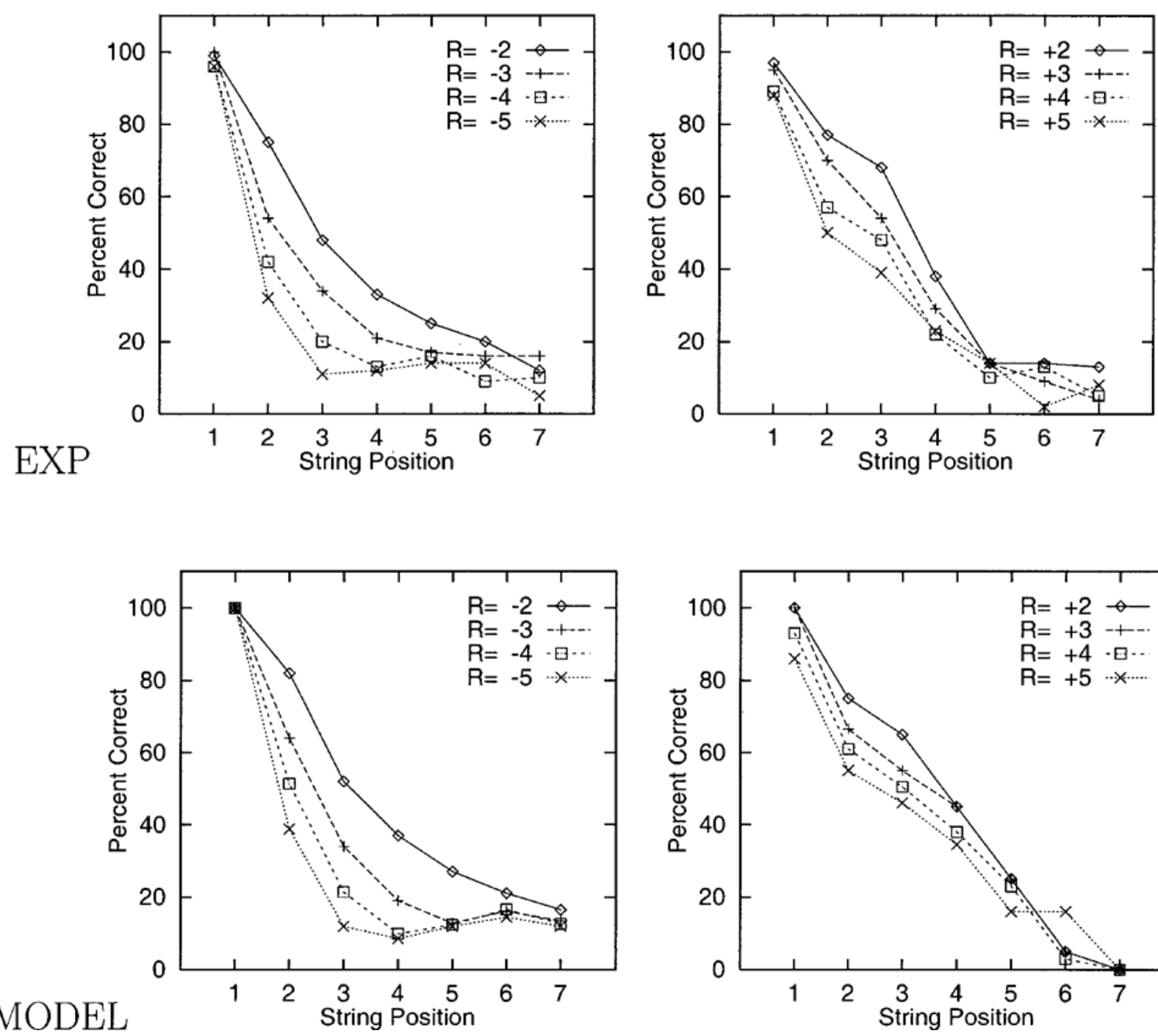

$\mathrm{RVF} / \mathrm{LH}$

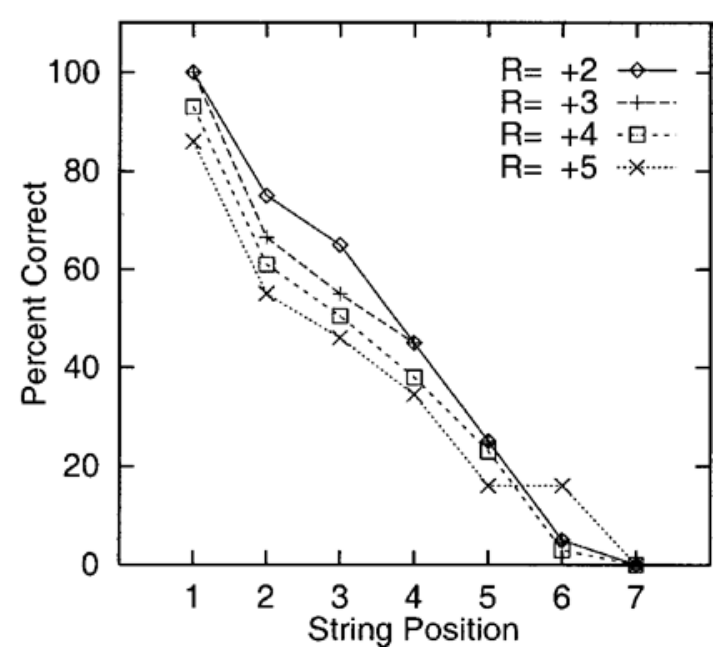

Figure 4. Experimental and modeled results for interaction of string position and retinal location from Wolford and Hollingsworth (1974). Graphs display percent correct at each string position for various retinal locations, $R$. The upper graphs display experimental data, and the lower graphs display the model's results. Graphs on the left display LVF/RH data, and graphs on the right display RVF/LH data.

\subsection{Costs of Transfer and Inversion}

In our specification of locational gradient formation, scanning direction determines the hemisphere for which acuity gradient inversion occurs. That is, inversion should occur for the visual field in which the slope of the acuity gradient is increasing with increasing string position. For languages scanned from left to right, this is the LVF/RH, as previously discussed. For languages read from right to left, inversion should occur in the opposite visual field, the $\mathrm{RVF} / \mathrm{LH}$. The other directional factor involved in locational gradient formation is callosal transfer, which is determined by which hemisphere is dominant for language. For the typical brain organization, transfer occurs from the
RH to the LH. For the atypical organization, transfer occurs in the opposite direction, from the LH to the RH.

We will refer to the process of inverting the slope of the acuity gradient as inversion, and we will refer to the process of callosal transfer as transfer. These two processes are independent of each other. We assume that each incurs some processing delay. We propose that transfer imposes a constant delay (independent of the number of letters transferred), whereas inversion incurs a delay that increases with the number of letters involved. We next discuss how this view is consistent with a variety of studies in which visual field of presentation, reading direction, and hemispheric dominance are varied. 
It has been found that an OVP exists for words that differs from the effects of acuity alone. In French and English, words are most easily perceived when fixation falls approximately one character to the left of the word's center (i.e., so that more of the word appears in the RVF than in the LVF; see Brysbaert, 1994; O'Regan et al., 1984). Presumably, the main determinant of the OVP is acuity; fixation at the word's center minimizes the loss of acuity at the retinal level (i.e., maximizes the minimal acuity). This implies that the leftward bias arises at some higher level of processing (Brysbaert et al., 1996; O'Regan et al., 1984).

O'Regan et al. (1984) proposed that the rightward shift occurs because the initial part of a word is usually the most useful in distinguishing the word from other words. They investigated the influence of locus of maximal information on OVP by varying the lexical structure of the word stimuli. Under fixation near the end of the word, words having unique combinations of letters at the end were more quickly processed than were words with unique letters at the beginning. Thus, they demonstrated that informational content does influence OVP. However, lexical structure cannot account for the entire OVP effect, because the relative advantage for information at the beginning is much greater than for information at the end. That is, the advantage for fixating on the information-rich part of the word (versus fixating on the opposite part of the word) is much greater if that part is at the beginning of the word than if it is at the end. Thus, it appears that an additional process is at work in determining the OVP.

For words presented entirely within a single visual field, a robust $\mathrm{RVF} / \mathrm{LH}$ advantage (relative to the LVF/ $\mathrm{RH}$ ) in both reaction time and accuracy has been found (for reviews, see Bryden, 1982, and Hellige, 1993). Brysbaert et al. (1996) have shown that there is a continuum of advantage for the RVF/LH as foveal presentation (involving both visual fields) changes to parafoveal presentation (involving a single visual field). This result implies that the leftward bias in the OVP and the hemifield RVF/ LH advantage both arise from the same underlying mechanism, as argued by Brysbaert et al. (1996).

What are these mechanisms that are responsible for this RVF/LH superiority? Various proposals have focused on different factors. Since information in the RVF is sent directly to the usually dominant LH, the advantage may arise from hemispheric factors. Alternatively, it may be associated with the scanning direction employed for a specific language, or it may depend on the position of distinguishing information within a word (Brysbaert et al., 1996; O'Regan et al., 1984). In addition to any informational influences, we propose that the effect depends on an interplay of both scanning and hemispheric influences. Namely, for presentation in the RVF/LH, neither inversion nor transfer is required for locational gradient formation, whereas, for presentation in the LVF/RH, both processes are necessary. Thus, the RVF has a large advantage over the LVF because less processing is required.

3.2.1. Right-to-left readers. Our proposal predicts that this RVF advantage should be reduced for a lan- guage read from right to left, because inversion becomes necessary for RVF/LH presentation and is no longer necessary for LVF/RH presentation. This prediction is consistent with current studies on reading direction and hemifield superiority (in the following discussion, subjects are assumed to be LH dominant). Previously, such experiments were designed to detect only the presence or absence of RVF/LH superiority. It was noted that an RVF/LH advantage does exist in Hebrew, and researchers concluded that reading direction did not affect visual field superiority (Carmon, Nachson, Isseroff, \& Starinsky, 1976; Faust, Kravetz, \& Babkoff, 1993). Because we propose that the RVF/LH superiority arises from the interplay of hemispheric dominance and reading direction, we suggest that it is important to consider the relative sizes of RVF/LH superiorities by reading direction.

Indeed, in a recent study of Farsi (a right-to-left language) versus English designed to study this issue (Melamed \& Zaidel, 1993), the RVF/LH advantage in Farsi was significantly smaller than in English for a word naming task. In a lexical decision task (i.e., where processing is dominated by letter encoding and lexical access, rather than phonological encoding), there was no RVF/LH advantage for Farsi. This combination of results led the authors to conclude that the increased RH contribution in Farsi (relative to English) selectively involves the visual input processing aspect of word recognition. Since inversion is a component of visual input processing, this is consistent with our suggestion that the RVF/LH advantage is reduced for languages scanned from right to left as a result of the cost of inversion in the RVF/LH. The results of an OVP study in Arabic (another right-to-left language) are also consistent with this proposal. In Arabic, the OVP is located at the center of the word, with no leftward bias (Farid \& Grainger, 1996). We suggest that the rightward shift of the OVP in Arabic (relative to left-toright languages) arises from the same underlying mechanisms as the reduced (or obliterated) RVF/LH advantage in Farsi-namely, that the cost of acuity gradient inversion is no longer incurred in the LVF/RH.

3.2.2. RH-dominant readers. Our proposal also predicts that the RVF/LH advantage should be reduced for RH-dominant, left-to-right readers, because transfer becomes necessary, whereas for LVF/RH presentation, transfer is no longer necessary. The results of the following experiment are consistent with this prediction and allow us to quantify the relative costs of inversion and transfer using a mathematical model.

Brysbaert (1994) studied the effect of cerebral dominance and viewing position on French readers, whose hemispheric dominance was determined by a battery of laterality tests. Subjects were to name words, where fixation point and word length were varied. The word was presented with either its initial letter or its final letter occurring at the central fixation point. The reaction time data are displayed in the upper panels of graphs in Figure 5. LH-dominant subjects showed the usual strong RVF/LH advantage: For words of three, four, five, seven, and nine letters, reaction times were significantly shorter 


\section{LH DOM}
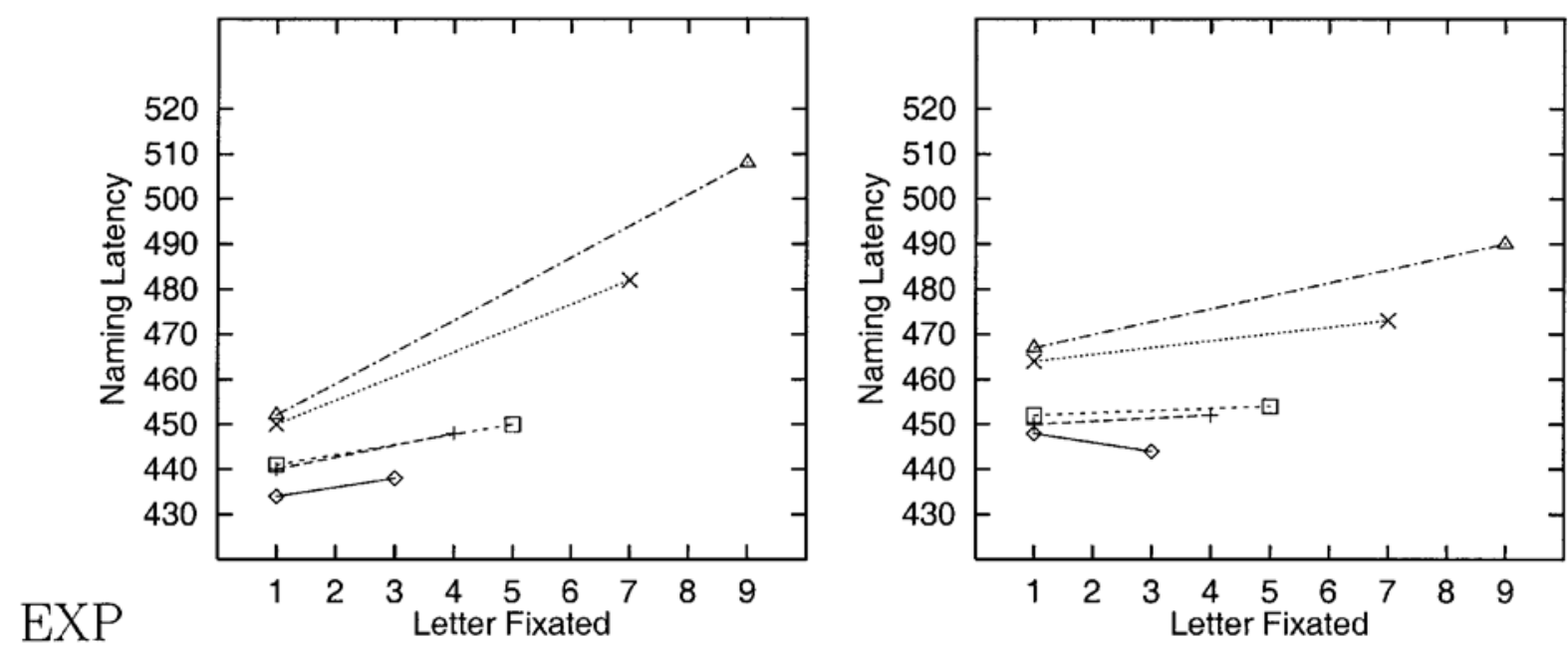

MODEL

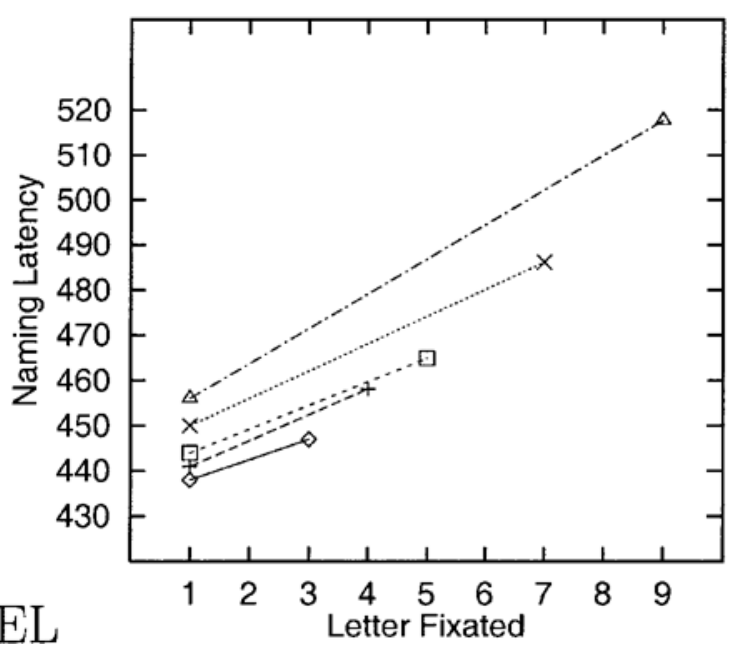

RH DOM

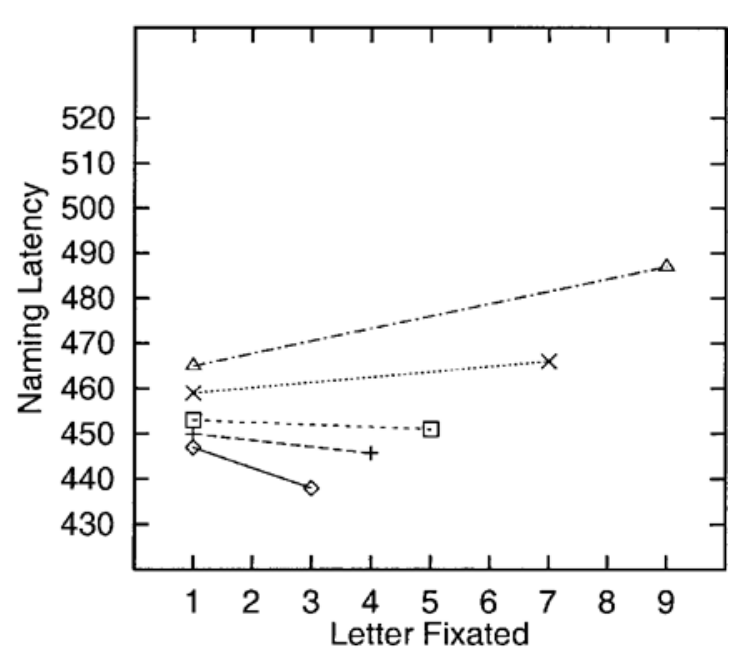

Figure 5. Experimental and modeled results for naming latency from Brysbaert (1994). Graphs display reaction times (in milliseconds) for initial- and final-letter fixations, where connected points represent words of the same length. Initial-letter fixation results in presentation to the $\mathrm{RVF} / \mathrm{LH}$, and final-letter fixation results in presentation to the $\mathrm{LVF} / \mathrm{RH}$. The upper graphs display experimental data, and the lower graphs display the modeled results. Graphs on the left display data for LH dominance, and graphs on the right display data for RH dominance.

for initial-letter fixation (i.e., word in RVF/LH) than for final-letter fixation (i.e., word in LVF/RH). The size of this difference increased with increasing word length, denoted as an increasing $R V F / L H$ advantage. In $\mathrm{RH}-$ dominant subjects, the RVF advantage was greatly reduced. For three-letter words, RH-dominant subjects had shorter reaction times for fixation on the final letter than on the initial letter (i.e., a trend toward a LVF/RH advantage). For four- and five-letter words, there was no difference between initial- and final-letter fixation. For the longer words, RH-dominant subjects, like LH-dominant subjects, displayed shorter reaction times for initial fix- ation than for final fixation. However, the size of the difference was much smaller in the RH-dominant subjects than in the LH-dominant subjects.

Brysbaert (1994) has argued that these data are consistent with the proposal that interhemispheric transfer is necessary for information falling in the visual field that projects to the nondominanthemisphere. That is, for $\mathrm{LH}$ dominant readers, information in the LVF/RH must undergo callosal transfer, and, for RH-dominant readers, information in the RVF/LH must undergo transfer. For LH-dominant readers, the shift from initial-letterfixation (word in RVF/LH) to final-letter fixation (word in LVF/ 
RH) exacts a large cost in naming latency, because interhemispheric transfer becomes necessary. However, for $\mathrm{RH}$-dominant readers, the shift from initial-letter to final-letter fixation removes the necessity of interhemispheric transfer, resulting in a greatly reduced cost for such a shift, relative to that for LH-dominant subjects. Presumably, the fact that the two patterns were not mirror images of each other (i.e., for most word lengths, there was no $\mathrm{LVF} / \mathrm{RH}$ advantage for $\mathrm{RH}$-dominant readers) is related to factors other than hemispheric dominance, such as reading direction (Brysbaert, 1994), as is consistent with our proposal.

On the basis of the increasing RVF/LH advantage with word length, Brysbaert (1994) proposed that the time required for interhemispheric transfer depends on the number of letters to be transferred. That is, because differences in latencies between final-letter fixation and initial-letter fixation increased as word length increased, Brysbaert concluded that callosal transfer time increases as the number of letters to be transferred increases. However, this interpretation is not fully consistent with the data. If transfer time depends on the number of letters being transferred, the difference in initial-letter fixation latencies for RH-dominant and LH-dominant readers should also increase with increasing word length. However, this difference does not increase; it is constant at about $15 \mathrm{msec}$ for all word lengths.

Our interpretation of this data is that interhemispheric transfer time is constant, whereas the time required to invert the acuity gradient increases with the number of letters involved. Such an assumption accounts for all aspects of the data. Because the time cost of inversion increases with the number of letters to invert, both hemispheres show an increasing RVF/LH advantage with word length. Because inversion is necessitated by $\mathrm{LVF} / \mathrm{RH}$ presentation regardless of dominant hemisphere, both hemispheres show an RVF/LH advantage for long words. However, since LVF/RH presentation to LH-dominant readers involves both acuity gradient inversion and hemispheric transfer (whereas only inversion is involved for LHdominant readers), the size of the $\mathrm{RVF} / \mathrm{LH}$ advantage is larger for LH-dominant readers. For RVF/LH presentation to RH-dominant readers, a constant interhemispheric transfer time is involved with no inversion time cost, accounting for the constant difference in initial-letter fixation times across dominant hemispheres. Possible processes underlying this proposed increasing inversion time will be discussed below.

On the basis of these ideas, we have developed a mathematical model of these naming data. The results of a fit of this mathematical model are displayed in the lower panels of Figure 5. The details of the model are given in Appendix B. Note that the model captures the salient aspects of the data. For final-letter fixation in LH-dominant readers, reaction times increase steeply with increasing length. For RH-dominant readers, the cost of final-letter fixation is not as steep. In fact, for three-letter words, a trend toward an LVF/RH advantage for RH-dominant readers appears. However, as word length increases, an $\mathrm{RVF} / \mathrm{LH}$ advantage emerges.

3.2.3. Time cost of inversion. It seems reasonable to assume that the time required for inversion increases with word length. Consider fixation on the final letter of a word. The amount by which the activations of this final letter's features must be reduced (in forming the locational gradient) depends on the number of features (letters) to its left. That is, a longer word results in a greater reduction in activation than does a shorter word. If it does take longer to accomplish a larger reduction, it would indeed be the case that the time cost of inversion increases with the number of letters involved (word length). However, increasing processing time with increasing length is usually taken as a sign of serial processing. How can this length-dependent process be reconciled with the assumption of parallel processing at the feature level? More specifically, parallel processing would imply that the features of the letter at fixation receive more inhibition (over the same time period) as the number of features to the left increases, implying that activation reduction time should be constant with increasing length. However, this reasoning depends on the assumption that increasing amounts of inhibitory input can be processed in the same amount of time. If it takes longer for incoming inhibition to take effect as the amount of that inhibition increases, a length effect would indeed emerge. Thus, a length effect for inversion is not necessarily contrary to the assumption of parallel inputs to feature nodes. This scenario is consistent with a theoretical analysis showing that a parallel process with exponential processing rates can mimic a serial system (Townsend, 1971).

If the increasing RVF/LH advantage does result from the necessity of acuity gradient inversion in the LVF/RH, this effect should disappear for a right-to-left language, since inversion is necessary for RVF/LH presentation, and not for LVF/RH presentation. The results of a study of Hebrew readers are consistent with this prediction. In a visual hemifield study of right-handed (presumably LHdominant) subjects who named Hebrew words of two to five characters, latency increased with word length (as would be expected due to the cost of phonological encoding), but this effect was independent of visual field (Koriat, 1985). That is, there was no increasing advantage of $\mathrm{RVF} / \mathrm{LH}$ presentation versus $\mathrm{LVF} / \mathrm{RH}$ presentation.

However, since we propose that inversion underlies the increasingly large RVF/LH advantage in a left-to-right language, why then was there no increasingly large LVF/RH advantage observed in the Koriat (1985) study? We suggest that the answer depends on the length of the Hebrew words used, coupled with the fact that RVF/LH presentation of Hebrew incurs inversion only, rather than both transfer and inversion. We propose that the time cost of inversion without transfer for five or fewer letters is small and is roughly equal to the time cost of transfer. This is consistent with the analogous results from Brysbaert (1994) for RH-dominant individuals (i.e., the mirrorimage case of LH-dominant, right-to-left readers), where 
$\mathrm{LVF} / \mathrm{RH}$ and $\mathrm{RVF} / \mathrm{LH}$ presentation latencies were roughly equivalent to each other for words of five or fewer letters; an $\mathrm{RVF} / \mathrm{LH}$ advantage for $\mathrm{RH}$-dominant, left-to-right readers emerged only for words longer than five letters, where, according to our model, the time cost of inversion became larger than the time cost of transfer. Analogously, we would expect a LVF/RH advantage for LHdominant, right-to-left readers to emerge only for words longer than five letters; such words were not included in the Koriat (1985) experiment.

Thus, our proposal implies that, in order to obtain a consistent LVF/RH advantage, subjects would have to be $\mathrm{RH}$-dominant, right-to-left readers. Indeed, in a study of Hebrew readers, left-handed subjects showed an LVF/RH advantage, whereas right-handed subjects did not (Orbach, 1967). Thus, a theory based on the time cost of interhemispheric transfer and acuity gradient inversion is consistent with the results of a range of studies contrasting dominant hemispheres, reading direction, and word lengths.

3.2.4. Position-specific reading deficit. Such a theory can also account for an unusual pattern of errors observed in several patients with LH damage (Costello \& Warrington, 1987; Cubelli, Nichelli, Bonito, De Tanti, \& Inzaghi, 1991; Katz \& Sevush, 1989). These patients suffered from parietal/occipital lesions that caused visual neglect or extinction on the right side of space, with normal perception on the left side. However, this pattern was reversed when reading words. Under central fixation, these patients tended to make errors that involved the initial letter (from the left side of space), while correctly retaining the other letters. They did not have any other language problems. This pattern of dysfunction was taken to support the notion of position-specific letter detectors. It was hypothesized that patients suffered from damage to letter detectors representing the first position (Katz \& Sevush, 1989).

We offer an alternative explanation for this error pattern, based on callosal transfer. We propose that visual feature information that is sent from the RH (i.e., representing the first half of the word) is not correctly received in the LH during locational gradient formation, as a result of the lesion. Because the initial letter has the lowest acuity, it is the most prone to degradation. (Although we propose that the activation of the first letter's features are elevated during locational gradient formation, this process cannot insert information that is not already there; it is like magnifying a noisy picture.) Thus, we propose that the lesion interferes with the callosal transfer of information pertaining to the first part of the word, where the first letter is the most vulnerable.

This analysis is consistent with several aspects of the patients' profiles. In their reading errors, patients tended to substitute, rather than omit, the initial letters, reflecting a misinterpretation of available information. When words were presented entirely in the RVF/LH to the patient described by Katz and Sevush (1989), errors were no more likely on the initial letter than on the other letters. Thus, when the initial letter had the highest acuity and did not have to undergo callosal transfer, it was no longer the most vulnerable. The location of the patients' lesions in posterior LH regions close to the visual areas is consistent with our proposal of impaired callosal transfer of visual information. In contrast, in Section 4.2, we discuss how the SERIOL framework can account for the behavior of patients having more anterior/central LH lesions, who show no frank spatial neglect, have generalized language problems, and make errors on the final letters of words.

\subsection{Hemispheric Modes of Processing}

We believe that our discussion of the roles of the hemispheres in locational gradient formation is relevant to current debates concerning supposed differing hemispheric "modes of processing" in reading. On the basis of visual hemifield data, researchers have proposed that string processing operates in parallel in the LH and serially in the RH (the studies described here were performed with left-to-right readers, unless specified otherwise). For example, normal subjects show no length effect in lexical decision for three- to six-letter words presented to the RVF/LH. However, for words presented to the $\mathrm{LVF} / \mathrm{RH}$, each additional letter increases reaction time by approximately $20 \mathrm{msec}$ (Ellis, Young, \& Anderson, 1988; Young \& Ellis, 1985). We suggest that it is not the case that the two hemispheres utilize different modes of processing, but rather that length effects arise with $\mathrm{LVF} / \mathrm{RH}$ presentation due to the cost of acuity gradient inversion, as discussed above.

A similar pattern of results emerged in a study of a callosotomized patient (Reuter-Lorenz \& Baynes, 1992). Lexical decision reaction times for words of three to five letters were constant for RVF/LH presentation. However, for $\mathrm{LVF} / \mathrm{RH}$ presentation, reaction times were $100 \mathrm{msec}$ longer for five-letter words than for three- or four-letter words (which were equivalent). These results were taken to be consistent with those of normal subjects and to be further evidence of different modes of processing across the hemispheres. However, we propose that the two length effects arose from different underlying processes. Note the difference in time scale between normals and the patient. For normals, each letter invoked a cost on the scale of $10 \mathrm{msec}$, as is consistent with our above mathematical model of the cost of interhemispheric transfer and acuity gradient inversion. However, the increase of $100 \mathrm{msec}$ for the patient is not on this scale; rather, it is on the scale of the proposed oscillatory cycle. Due to the destruction of the callosum, the patient would have to process words presented to the LVF/RH entirely within the RH. We propose that letter nodes in the RH are more slowly activated than those in the LH, so strings of letters that are normally represented in one oscillatory cycle in the $\mathrm{LH}$ require an additional oscillatory cycle in the RH. This proposal is consistent with the observation that there was a sharp discontinuity in the patient's reaction times (i.e., 0 -msec difference between three- and four-letter words, and 100msec difference bewteen four- and five-letter words), as is consistent with going from one oscillatory cycle to two cycles. Also, letter priming experiments with the patient 
showed greatly reduced facilitation in the $\mathrm{RH}$ relative to the $\mathrm{LH}$, as is consistent with the proposal of inadequate activation of letter nodes in the RH.

In summary, we propose that different factors underlie the length effects for LVF/RH presentation observed in normals and in the patient. For normals, it arises at the feature level from processes underlying the formation of the locational gradient. For the patient, it arises at the letter level, as a direct result of the proposed temporal basis of letter position coding. (In Section 4.1, we discuss why no length effect emerges directly from the temporal encoding at the letter level for normal subjects.)

Patterns of positional errors have also been taken as evidence for different hemispheric modes of processing (Hellige, Cowin, \& Eng, 1995; Hellige \& Scott, 1997; Marks \& Hellige, 1999). Subjects were to identify quickly displayed CVC trigrams presented in a vertical column, with each letter in the upright position. For LVF/RH presentation, subjects made many more errors involving the last letter than the first letter of the string. For RVF/LH presentation, this finding was greatly attenuated: There were more errors on the first letter and fewer errors on the last letter (relative to LVF/RH presentation), resulting in a more even distribution of errors across the string. There was also a strong effect of visual field on accuracy, with more total errors in the LVF/RH than in the RVF/LH. These patterns were taken to be evidence of parallel processing of strings by specialized linguistic modules in the $\mathrm{LH}$ and less efficient, serial processing in the RH.

However, a counterintuitive result arises when input is directed to both hemispheres simultaneously. When stimuli are presented bilaterally, the error pattern that emerges is more similar to the $\mathrm{LVF} / \mathrm{RH}$ pattern than to the $\mathrm{RVF} / \mathrm{LH}$ pattern (Marks \& Hellige, 1999). Thus, even though the LH is more effective than the RH at performing the task, the RH's mode of processing (i.e., error pattern) seems to dominate when the stimuli are presented to both hemispheres simultaneously.

On the basis of locational gradient formation, we offer an explanation of these results that accounts for both the asymmetry of the error patterns and the bilateral pattern. Despite the unorthodox vertical format, we assume that encoding processes similar to those used for horizontal presentation were invoked. Hellige and colleagues (Hellige et al., 1995; Hellige \& Scott, 1997; Marks \& Hellige, 1999), in their analyses, also assume that the data from these experiments are relevant to normal string processing. More specifically, we assume that the elements of the string are mentally rotated to the canonical horizontal position, and, then, the rotated image is processed as usual. This mental rotation assumption is consistent with the finding that reaction time for lexical decision increases smoothly with rotation angle for two-letter strings (Koriat \& Norman, 1985). (For longer strings, reaction time does not increase smoothly with angle of rotation. We have shown that this effect could result from mental rotation progressively degrading inputs to the letter level, requiring increasing numbers of oscillatory cycles to represent the string. See Whitney, in press.)
The proposed inhibitory processing underlying locational gradient formation can acount for the differing hemispheric error patterns. As discussed above, in the hemisphere in which inversion occurs, the acuity gradient is transformed in the locational gradient by strong excitation to the initial letter, coupled with strong directional inhibition. This transformation could result in a steeper partial locational gradient in that hemisphere. Thus, for English readers, the partial locational gradient could be steeper for the LVF/RH than for the RVF/LH. That is, the inputs to the first letter in each hemisphere are similar, whereas the input to the final letter in the LVF/RH is lower than in the RVF/LH (see Figure 3, lower left panel). This accounts for the greater percentage of errors on the final letter in the LVF/RH.

Following callosal transfer, the RH features inhibit the LH features to form a monotonically decreasing locational gradient. We propose that, under bilateral stimulation, the error pattern of the $\mathrm{RH}$ predominates because the LVF/RH features inhibit the RVF/LH features. This is not inconsistent with the finding that recognition is better in the $\mathrm{LH} / \mathrm{RVF}$ than in the RH/LVF with respect to presentation to a single visual field; recognition is better in the RVF/LH because there is no degradation due to callosal transfer. However, when both visual fields receive input, we propose that input from the LVF/RH dominates due to the way in which hemispheric representations of letter strings are normally integrated.

This analysis implies that the asymmetry of hemispheric error patterns should vary with reading direction. For languages read from right to left, the patterns should be reversed, since acuity gradient inversion should then occur in the RVF/LH. This is precisely the finding reported in a study of Hebrew readers performing the trigram identification task; for those readers, percentage of final-letter errors was greater in the RVF/LH than in the LVF/RH, and the bilateral pattern was the same as that of the RVF/LH (Eviatar, 1999). For languages that are read from top to bottom, there should be no hemispheric asymmetry, since acuity gradient inversion should occur along the vertical axis, not the horizontal axis. Indeed, a study of Japanese kana, for which the vertical orientation is normal, showed no differences between LVF/RH, RVF/LH, and bilateral patterns (Hellige \& Yamauchi, 1999). Thus, these findings are inconsistent with a hemispheric specialization account but are predicted by the locational gradient account.

This type of experiment has also been performed with the trigrams presented horizontally rather than vertically (Eviatar, 1999; Hellige \& Cowin, 1996), yielding conflicting results. Eviatar reports abolition of the the hemispheric asymmetries for both English and Hebrew, whereas Hellige and Cowin report that the asymmetry for English is maintained. These findings can also be accounted for within the SERIOL framework. For vertical presentation, the resulting mentally rotated image has no acuity gradient, because all letters were originally presented at essentially the same distance from fixation. In the hemisphere in which inversion occurs, the steepness of the locational gradient is much greater than usual, be- 
cause there is no opposing acuity gradient. In the hemisphere in which inversion does not occur, the resulting locational gradient is shallower than usual, because there is no supporting acuity gradient. Thus, vertical presentation should accentuate differences in directional inhibition across the two hemispheres. For horizontal presentation, this asymmetry may or may not be evident, depending on the actual acuity gradients associated with the stimuli. The locations of the stimuli differed across the two experiments cited above. An analysis based on locational gradient formation, presented in Appendix A, showed that there should be more asymmetry for the location used by Hellige and Cowin than that used by Eviatar. So the difference in stimulus location can account for the difference in asymmetry across the experiments.

Thus, we conclude that the seemingly different modes of processing across the two hemispheres are not attributable to serial versus parallel processing. We propose that letter activations are always serial and that hemispheric differences stem from the direction of slope of the acuity gradient relative to that of the locational gradient.

\section{STUDIES RELEVANT TO LETTER LEVEL REPRESENTATION}

Now, we turn our attention to experimental data best addressed at the letter level of the SERIOL framework. Recall that the locational gradient, in conjunction with the dynamics of the letter level nodes, induces a temporal firing pattern across the letter nodes. As a consequence of this process, a positional activation gradient arises across the letter nodes. First, we discuss studies relevant to the temporal aspect of letter position coding. Then, we examine the activation pattern of the positional gradient.

\subsection{Temporal Encoding}

We propose that the encoding of letter position is based on sequential firing of letter nodes. As is consistent with the observation of nested oscillations recorded in the human cortex in cognitive processing (Llinas \& Ribary, 1993), we suggest that each letter position corresponds to a successive 25 -msec subcycle within an oscillatory period of $\approx 200 \mathrm{msec}$. This proposal is consistent with some curious results from a study involving sequential letter presentation. In that study, the letters of eight-letter words were presented one at a time across a horizontal row (Mewhort \& Beal, 1977). The interval between successive letters (interstimulus interval, ISI) was varied, and performance was measured as probability of correctly identifying the word. For ISIs of $0 \mathrm{msec}, 50 \mathrm{msec}$, and $125 \mathrm{msec}$, performance declined from $98 \%$ to $70 \%$ to $50 \%$, respectively. However, for an ISI of $250 \mathrm{msec}$, performance rebounded to $65 \%$, rather than continuing to fall off. Our interpretation of this result is that sequential letter presentation interfered with the normal phasic coding of letter position. Letter presentations were maximally out of phase with respect to normal at an ISI of $125 \mathrm{msec}$ (worst performance). Performance levels for $50 \mathrm{msec}$ and $250 \mathrm{msec}$ were similar, consistent with an oscillatory cycle length of $200 \mathrm{msec}$. This interpretation predicts that, when performance is impaired, the order of the letters should be perceived incorrectly due to presentation rate and internal encoding being out of phase. The subjects' reports for incorrectly identified words were consistent with this prediction: Detection of individual letters was not impaired at $125 \mathrm{msec}$ (the ISI of worst performance) relative to the other ISIs, whereas report of the preceived order of letters at $125 \mathrm{msec}$ was impaired relative to the other ISIs.

This temporal encoding hypothesis is countered by the results of lexical decision experiments, which have been interpreted as supporting strictly parallel processing of letter strings. Lexical decision tasks have shown that reaction times for words of three to six letters are equivalent under central fixation (Forster \& Chambers, 1973; Frederiksen \& Kroll, 1976; Green \& Shallice, 1976) and under RVF/LH presentation, as discussed above. Can a temporal, serial representation of letter position be reconciled with the lack of a word length effect? We suggest that this finding should not be thought of as indicating that six-letter words are recognized as quickly as threeletter words, but rather that three-letter words are as slow to be recognized as six-letter words. In other words, a minimum reaction time exists. This minimum reaction time is based on completion of an oscillatory cycle. Words that can be represented within one oscillatory cycle have similar reaction times.

As mentioned above, we propose that the final letter node of a string continues to fire until the end of the oscillatory cycle. We suggest that this mechanism is involved in distinguishing short words from longer words. It should be the case that the string "cat" activates the word node "CAT" more strongly than it activates the word node "CART." In our scheme, the letter node "T" becomes more active as a result of the presentation of "cat" than as a result of the presentation of "cart" because it starts firing earlier in the cycle. Assuming that the connection weights reflect this difference, the string "cat" will indeed activate the node "CAT" more than the node "CART" since the weights (via the bigrams) from " $T$ " to "CAT" are larger than from "T" to "CART." The longer the "T" letter node fires (i.e., the higher its activation), the larger the difference in activation levels between "CAT" and "CART" becomes. Sufficient information to distinguish a winner and inhibit other word contenders may not be available until the end of the oscillatory cycle. As a result, it takes as long to recognize three-letter words as it does to recognize six-letter words. So, we argue that the absence of a length effect does not necessarily indicate parallel processing. Thus, words that can be represented in a single cycle (i.e., of approximately seven letters or less) should have equivalent reaction times.

Thus, under normal reading conditions, the temporal nature of letter position coding is not evident. However, if artificial presentation conditions (such as sequential letter presentation) are introduced, then temporal effects can emerge. Strong length effects on reaction times for rotated letter strings have also been observed. For rotation 
angles of $120^{\circ}$ or more, each additional letter increases reaction time by $200 \mathrm{msec}$ (Koriat \& Norman, 1985). Note that this increase is exactly the length of the proposed oscillatory cycle. We have constructed a model based on encoding within oscillatory cycles that accurately accounts for the complex interaction of angle and length on lexical decision reaction time observed for rotated strings (Whitney, in press).

Thus, we suggest that sharp jumps in reaction times result when information that can normally be represented in a single cycle must be represented across multiple cycles due to degradation of the input. What about words that are too long to be represented in a single cycle? There is no evidence for a sharp jump in reaction times as word length increases above seven letters. Presumably, under normal conditions, a mechanism exists for composing information smoothly across cycles. That mechanism is beyond the scope of this paper.

\subsection{Positional Gradient}

We have proposed that the varying levels of input to letter nodes that serve to induce the temporal firing pattern also create a positional activation gradient across the letter nodes. We believe that this mechanism can account for conflicting findings regarding the perceptibility of strings' final letters. In general, the final letter has been found to be preferentially perceived with respect to the internal letters, denoted a final-letter advantage (Hammond \& Green, 1982; Lefton et al., 1978; Mason, 1982; Perea, 1998). However, in other studies the final letter was the least well perceived of all the letters (Hellige et al., 1995; error data analysis in Humphreys et al., 1990; five- and six-letter words in Montant et al., 1998). In the SERIOL framework, the activation level of the node representing the final letter is sensitive to when in the cycle it starts to fire. Recall that a letter node continues to fire until it is inhibited by another letter node. However, the final letter node does not undergo such inhibition and continues to fire until it cannot pass threshhold due to the down phase of the oscillatory cycle. If overall input levels to the letter nodes are low, the final letter node cannot start firing until late in the cycle, and, therefore, it attains only low level of activation. If overall input levels are high, the final letter node can start firing earlier in the cycle and can reach a level of activation that exceeds that of the interior letters.

We propose that a very short presentation duration results in such a low overall level of input, whereas normal presentation conditions correspond to higher input levels that give a final-letter advantage. This proposal explains the above experimental findings: All those experiments in which a final-letter advantage did occur involved presentation durations of $75 \mathrm{msec}$ or more, whereas all those experiments in which a final-letter advantage did not occur involved presentation durations of $50 \mathrm{msec}$ or less. It also explains the finding in Eviatar (1999) that, for target letter detection within a trigram, decreasing exposure duration from $80 \mathrm{msec}$ to $40 \mathrm{msec}$ increases misses for targets in the final position but does not increase misses in the initial position.
Studies with brain-damaged patients have also demonstrated difficulties with the final letter of strings. In a study of a group of patients with LH damage who have language problems and are prone to reading errors, the final letter of a word was the letter least likely to be correctly retained in an error response. In fact, the probability of retention in the error response monotonically decreased as letter position within the word increased (Berndt \& Haendiges, 1997; Whitney \& Berndt, 1999). This pattern of errors is easily accounted for within the SERIOL framework by making two assumptions: (1) The excitability of letter nodes is reduced, pushing the firing of the letter nodes late into oscillatory cycle (as does a short presentation duration for normal subjects), negating any final-letter advantage, and (2) high levels of noise are incorporated into the word node activations. Because initial letters have the highest activations, word nodes that share initial letters with the target word are also significantly activated and are likely to be selected in the presence of noise. In simulations, these assumptions allowed replication of various aspects of the patients' error patterns (Whitney \& Berndt, 1999).

Montant et al. (1998) studied a patient, C.P., with pure alexia. C.P. suffered LH damage and showed an unusual OVP, where word recognition was optimal when fixation occurred near the last letter of the word (so that the word appeared mostly in the LVF). On the basis of a series of experiments, the authors observed that C.P. suffered from poor perceptual processing of letter stimuli in the RVF/ LH. However, this finding did not fully account for C.P.'s performance, since processing of words' final letters was always significantly impaired, independently of visual field of presentation. In experiments with normal subjects using very short presentation durations, the authors also found a pattern of reduced accuracy in processing the final letters of words.

On the basis of these data, the authors concluded that C.P.'s performance reflected an exaggeration of normal aspects of word processing. They suggested that C.P.'s dysfunction had uncovered a "prelexical level of word processing, where letter information is weighted differently as a function of letter position in a word-centered space" (Montant et al., 1998, p. 123). This conclusion is quite similar to our proposed positional gradient. However, the authors attribute this weighting to a different source-namely, perceptual learning. That is, they proposed that "the reading system weights letter units as a function of the quality of available visual information" (Montant et al., 1998, p. 125). However, we think that this account is not consistent with the data. If the weighting is perceptually based, it should have roughly the same shape as the acuity gradient: highest at fixation, and lower at the word boundaries. However, this is not the observed pattern; perceptibility is highest at the initial letter and generally falls off from left to right.

Rather, as is consistent with the observed pattern, we propose a more significant role for the apparent weighting of letter units, as arising from mechanisms directly involved in the encoding of letter position. These mech- 
anisms override the effects of perception, inverting the acuity gradient to form a locational gradient that induces a temporal encoding of letter position, as well as a positional activation gradient. This positional gradient has roughly the same shape as the locational gradient.

Although, for normal subjects, letter perceptibility falls off from left to right (with the exception of the final position) for strings presented entirely within a single visual field (Lefton et al., 1978), this monotonicity is not maintained if the string is presented centrally. In that case, a $\mathrm{W}$-shaped pattern of perceptibility emerges, with increased perceptibility for the letter under fixation (in addition to the initial and final letters; see Hammond \& Green, 1982; Lefton et al., 1978; Mason, 1982). This result is not inconsistent with our model. Such a pattern of activation can arise at the letter level in our model if the slope of the locational gradient is not smooth near the fixated letter. More precisely, this can occur if the amount of input reaching the fixated letter is only slightly less than that of the previous letter but much greater than that of the next letter. The firing of the previous letter is truncated by the firing of the fixated letter, whereas the fixated letter fires for an extended period until it is inhibited by the next letter. This scenario results in a higher level of activation for the fixated letter than for its neighbors. Because we propose that the two partial hemispheric locational gradients are joined at the letter under fixation, such a lack of smoothness is plausible (see Figure 3, bottom right panel).

\section{STUDIES OF LETTER POSITION ENCODING}

We now turn to recent experiments specifically designed to investigate the nature of letter position encoding in normal readers (Grainger \& Jacobs, 1991; Humphreys et al., 1990; Peressotti \& Grainger, 1995, 1999). First, we give an overview of these experiments; then, we discuss how the SERIOL framework accounts for these results.

\subsection{Results of Position Encoding Experiments}

Some of the studies involved nonword letter strings in order to eliminate top-down influences, whereas others involved words. First, we will discuss those utilizing nonword strings. Grainger and Jacobs (1991) asked subjects to perform an alphabetic decision task, in which they were to identify whether or not a character embedded in a string of hash marks was a letter. The target character appeared in either the initial or the terminal position of a fivecharacter test string (e.g., "\#\#\#\#T" or “T\#\#\#\#”). The test string was preceded by a prime string, which was a neutral string ("xxxxx"), a five-letter word containing the target letter, or a target letter embedded in "x"s. For both types of primes, the target letter could appear in the same position as in the test string (e.g., prime string " $x \times x x T$ " for test string “\#\#\#\#T") or in the opposite position (crossposition case; e.g., prime string "TABLE" for test string "\#\#\#\#T"). In addition, the presentation duration of the prime string was varied (16 vs. $64 \mathrm{msec})$. Priming was measured as decrease in reaction time (with respect to a neutral prime) in identifying the target letter. In order to ensure that facilitatory effects of prime presentation did not result from physical overlap, the target letter in the prime and test strings occupied different absolute locations on the screen.

The results showed priming for most of the sameposition cases. There was also evidence for priming in one of the cross-position cases (64 msec, nonword, target letter in the initial position of the prime string). These experiments indicate that same-position priming is more robust than cross-position priming but that cross-position priming can occur. The following experiments provide futher evidence for cross-position priming.

Peressotti and Grainger (1995) also asked subjects to perform an alphabetic decision task, in which they were to determine whether or not strings of three characters consisted solely of letters (e.g., "TBR" vs. “TB\$”). Primes were also trigrams, consisting of characters from the test string, either in the same order (same-position cases) or in a different order (cross-position cases). The presentation duration of the prime string was varied $(33,50$, and $66 \mathrm{msec}$ ). Performance was measured as response time to the test string. In order to ensure that facilitation did not result from physical overlap, prime strings and test strings were presented in different-sized fonts.

Overall, the results of Peressotti and Grainger (1995) are similar to those of Grainger and Jacobs (1991). Crossposition priming occurred, but only when prime exposure durations were at least $50 \mathrm{msec}$. Same-position priming was more robust, occurring even at shorter prime durations and giving higher levels of facilitation.

The next pair of studies involved word recognition. In Humphreys et al. (1990), subjects were to identify words that were presented briefly $(40 \mathrm{msec})$, such that recognition levels were not at ceiling. Performance was measured as percentage of words correctly identified. Primes were nonwords in which the positions of letters in common with the test word were manipulated. Primes were presented in lowercase, and test words were presented in uppercase.

For primes composed of the same letters as the test word but rearranged into a different order, no facilitation was observed. Thus, no cross-position priming was observed for test words. For primes having letters in the same order as the test word but in different absolute positions, facilitation was observed. For example, the prime "okte" enhanced recognition of the test word "WHITE," even though the common letters "TE" were at positions 3 and 4 in the prime string but at positions 4 and 5 in the test word. We will designate this result as relative-position priming, because letter order and word boundaries of a portion of the prime string were the same as the test word.

These same results (lack of cross-position priming and occurrence of relative-position priming) were also observed by Peresotti and Grainger (1999). In those experiments, subjects performed lexical decision on six-letter strings, and performance was measured as response time. Primes and test strings were in fonts of different sizes. At exposure durations of $50 \mathrm{msec}$, four-letter primes con- 
sisting of the first, third, fourth, and sixth letters of the test string in that order were facilitatory (e.g., "BLCN" for the French word "BALCON"), whereas primes consisting of those letters in a different order were not. Primes incorporating space fillers among the letters to preserve absolute position (e.g., "B_LC_N") were no more facilitatory than primes without space fillers.

The results of Humphreys et al. (1990) and Peressotti and Grainger (1999) are similar, indicating that, for word test strings, relative-position, but not cross-position, priming occurs. Although the prime durations employed in Humphreys et al. (durations of $\approx 40 \mathrm{msec}$ ) were somewhat shorter than those that invoked cross-position priming for nonwords in Peressotti and Grainger (1995) (durations of 50 and $66 \mathrm{msec}$ ), the results of Peressotti and Grainger (1999) indicate that cross-position priming does not occur for word test strings even at 50-msec durations. Thus, it appears that the absence of cross-position priming in these word-based experiments arises from the nature of the test string (i.e., word vs. nonword), rather than from the prime exposure duration.

In summary, the results of the above four studies indicate that cross-position priming can occur under the proper conditions: when prime exposure duration $\geq 50 \mathrm{msec}$, and both prime and test strings are not words. For word test strings, relative-position priming is as effective as sameposition priming.

\subsection{The SERIOL Framework and Position Encoding Experiments}

What do these results imply about the nature of letter order encoding? The fact that relative-position priming occurs when absolute position is violated indicates that some representation other than a channel-specific coding scheme is involved. These results are consistent with a context-unitcoding scheme. However, the fact that priming of individual letters occurs suggests that activation of context units requires activation of constituent letters. These results are consistent with a scheme in which letter units activate context units, as we propose. The existence of separate letter and bigram levels in the framework provides a mechanism for the activation of context units by constituent letters and thus accounts for individual letter priming and relative-position priming in word test strings. In the above experiments, cross-position priming was found to occur for nonword test strings, but not for word test strings. This result is consistent with the assumption that the activation of bigram nodes, rather than of letter nodes, dominates the efficacy of priming for word test strings. Cross-position primes do not activate the proper bigram nodes, so facilitation does not occur for word test strings. However, nonword test strings isolate activations at the letter level, revealing cross-position priming.

Could the initial encoding of letters be channel specific? The above experiments have demonstrated that same-position priming is quite robust, as is consistent with a channel-specific model. However, since crossposition priming can occur, a channel-specific model is not sufficient. In order to accommodate this finding, Peressotti and Grainger (1995) proposed a two-level model consisting of channel-specific letter units and positionindependent letter units.

Our framework incorporates a single set of letter nodes (as opposed to separate sets for each position, as in channelspecific coding), where position is tagged by timing of firing. Since different positions can be represented by the same letter node, cross-position priming at the letter level is possible. How then can we account for the robust same-position priming in the absence of channel-specific letter units within the SERIOL framework? We propose that the same-position results arose from the preorthographic level. Although the target letters appeared at different physical locations in the prime and test string presentations, we propose that interactions at the feature level could produce the appearance of position-specific priming, due to the proposed locational tuning of the feature nodes. On the basis of this hypothesis, we have developed mathematical models that replicate the experimental data (they are described in Whitney, 2001).

Thus, the SERIOL framework can account for the different types of priming observed in these experiments. We propose that same-position priming arises at the feature level, cross-position priming arises at the letter level, and relative-position priming arises at the bigram level. This analysis is consistent with the differing time courses and types of stimuli that evoked these types of priming.

\section{DISCUSSION}

As discussed in the introduction, the interpretation of experimental results showing decreasing letter perceptibility with increasing string position has changed over the years. Initially, it was assumed that these results emerged from a serial scan on an input trace (Lefton et al., 1978; Mewhort et al., 1969). Current accounts focus on perceptual factors and/or attention to the area of maximal information within the string, where maximal information is conditioned by top-down processes (Brysbaert et al., 1996; Montant et al., 1998; O’Regan et al., 1984).

We return to the earlier notion of a serial process. Our proposal that a locational gradient across letter features induces a temporal firing sequence is similar to a serial scan. However, we do not characterize this process as a "scan," because that term implies that some entity is shifting attention across a representation of the input string. Rather, we propose that the serial firing emerges from interactions between graded inputs, lateral inhibition, and subthreshhold oscillations. This account implies that the serial firing arises at a very low level of processing that does not correspond to attentional mechanisms.

Attention may play a role in acquiring the mechanisms that allow this process to take place automatically, just as conscious effort is required in learning any new skill before it becomes automatic. Once learned, however, this process is not subject to conscious, attentional control. This conclusion is consistent with various studies. In string matching experiments (Proctor \& Healy, 1987), the 
position of difference between two strings strongly influenced how long it took subjects to respond, despite the fact that position was irrelevant in determining the response. This was a very robust result, indicating that positional information is impossible to ignore and, thus, not under conscious control. In another study, subjects who had suffered right parietal damage (resulting in attentional deficits) were to read letter strings that were either words or pronounceable nonwords (Sieroff, Pollatsek, \& Posner, 1988). Subjects did not exhibit deficits when reading words, but they did when reading nonwords. This pattern of results was replicated in normal subjects using misdirected attentional cues to interfere with deployment of attention while reading (Sieroff \& Posner, 1988). These results were interpreted as indicating that top-down, conscious attention is not required to access the lexicon; however, when a letter string does not receive lexical support (i.e., is a nonword), attention is invoked in processing, revealing deficits or interference. Thus, these results are consistent with our proposal that top-down processes are not necessary for formation of the locational gradient (a prelexical process), and, therefore, attentional processes are not involved in the perceptual advantage for initial letters of strings.

We propose that the letter perceptibility pattern arises from a temporal encoding of letter position. Thus, we attribute a much more important role to the underlying source of positional effects than do current accounts. There is a level in the SERIOL framework that is roughly consistent with perceptual and attentional accountsnamely, the bigram-to-word connections. In that interface, connection weights correspond to the activations of bigrams constituting each word. Bigram activations depend on letter activations, which depend on letter position. Thus, weights are learned that correspond to letter activations, as proposed by a perceptual account. However, our proposed letter activations arise as a consequence of the letter position encoding process, not as a result of perceptual factors. As discussed above, the lack of congruity between the acuity gradient and the observed pattern of perceptibility is more consistent with our account than with a perceptual account.

The bigram node that is activated by the first and second letters of a word will have the highest connection weight to that word node. Thus, this bigram confers the most information about the word, as is similar to theories of attentional deployment to the locus of maximal information. However, the accounts of causality are reversed. Attentional accounts suggest that the initial letters of a word carry the most information due to the patterns of spelling in a language; therefore, weights on initial letters are high (Brysbaert et al., 1996; O'Regan et al., 1984). In contrast, we propose that weights on initial letters are high because their activations are elevated as a consequence of the levels of input necessary to induce them to fire early in the oscillatory cycle; therefore, initial letters carry the most information. Of course, both factors could be at work.
Although our framework shares some features of current accounts, the underlying assumption of serial encoding is contrary to many current formulations. What factors resulted in the abandonment of the serial-scan assumption? We conclude that it was due to an absence of a length effect in lexical decision experiments (Forster \& Chambers, 1973; Frederiksen \& Kroll, 1976; Green \& Shallice, 1976), coupled with the advent of the emphasis on parallel computation in connectionist modeling. In response to the former factor, we have argued above that if word recognition is unitized by an oscillatory cycle, then no length effect would emerge even if a string is represented by the serial firing of letter nodes. Thus, lack of a length effect does not necessarily imply purely parallel processing. The latter factor (emphasis on parallel computation) pertains to a current paradigm, not specifically to experimental evidence. In fact, in contrast to the rate-coding assumption underlying most parallel connectionist models, neurobiologists have recently concluded that timing of firing is important in informational encoding (Berry et al., 1997; de Ruyter van Steveninck et al., 1997; Reike et al., 1997; Victor \& Purpura, 1996).

The SERIOL framework traces the encoding and decoding of a temporal representation of letter position from the formation of a locational gradient at the feature level, to the induction of a serial firing pattern at the letter level, to the conversion to context coding at the bigram level, to the representation of entire words at the topmost level. Is such an elaborate scheme really necessary? Couldn't letter position be directly coded by an activation gradient across letter positions, without inducing a temporal firing pattern, and without bigrams?

We conclude that the answer to this question is "no." In an early computational version of our model, we represented letter position as an activation gradient (with no temporal aspect) across letter nodes, with letter nodes connecting directly to word nodes. Such a model was not very robust, and the types of errors that arose when the model was lesioned were not consistent with those produced by brain-damaged patients. The addition of the bigram level alleviated these problems (Whitney \& Berndt, 1999) and is consistent with evidence from normal readers on the importance of letter order (Humphreys et al., 1990; Peressotti \& Grainger, 1999). A letter level is necessary, in addition to the bigram level, to be consistent with experimental evidence on individual letter priming (Grainger \& Jacobs, 1991; Humphreys et al., 1990; Peressotti \& Grainger; 1995) and to provide a mechanism to activate the bigram nodes.

Why then is a temporal encoding necessary? Couldn't a positional gradient across letter nodes directly activate bigram nodes? One could postulate a bigram unit that is activated when its constituent letters have the correct relative activation levels (i.e., the first letter is more active than the second), rather than requring a specific order of firing. Recent neurobiological studies and theories have indicated that timing of firing of individual spikes is an important method of encoding information 
(Hopfield, 1995; Reike et al., 1997). If indeed the neural system operates in such a manner, comparing timing of firing, rather than rates of firing, is simpler and more precise. That is, the combination of firing rate (input level to a letter node) with the letter nodes' internal dynamics to create a temporal firing pattern results in a more precise representation of information than firing rate alone, and, therefore, neural systems can compare firing timings more accurately than firing rates. Additionally, all other modalities of language perception and production involve serial processing (i.e., speaking, listening, and writing), so it is plausible that a canonical temporal representation for language exists, into which visual input is converted.

Why then couldn't the temporal letter position encoding, with its strong representation of letter order, activate word nodes directly without bigram nodes? Such a scheme would require precise comparison of the relative timing of many inputs and could lead to errors. It is computationally more robust to compare the timing of pairs of inputs and to consolidate those results, as specified in the bigram and word levels of our framework.

Thus, we conclude that the functionality of each level of representation is necessary. As a whole, the SERIOL framework unifies and provides an account of many heretofore puzzling experimental results on reading: positional perceptibility of letters in strings, the paradox of the finalletter advantage/disadvantage, visual field differences in letter perceptibility, the location of the OVP and its relationship to reading direction, hemispheric modes of processing, and patterns of same-position, cross-position, and relative-position letter priming.

\section{REFERENCES}

Alpern, M. (1962). Introduction. In H. Dawson (Ed.), The eye (Vol. 3, pp. 3-5). New York: Academic Press.

Berndt, R. S., \& Haendiges, A. N. (1997). Positional effects in dyslexic "visual errors": Constraints on the interpretation of word substitutions. Brain \& Language, 60, 112-115.

Berry, M. J., Warland, D. K., \& Meister, M. (1997). The structure and precision of retinal spike trains. Proceedings of the National Academy of Sciences, 94, 5411-5416.

BJork, E. L., \& Murray, J. T. (1977). On the nature of input channels in visual processing. Psychological Review, 84, 472-484.

BRYDEN, M. P. (1982). Laterality: Functional asymmetry in the intact brain. New York: Academic Press.

BRYSBAERT, M. (1994). Interhemispheric transfer and the processing of foveally presented stimuli. Behavioural Brain Research, 64, 151-161.

Brysbaert, M., Vitu, F., \& Schroyens, W. (1996). The right visual field advantage and the optimal viewing position effect: On the relation between foveal and parafoveal word recognition. Neuropsychology, 10, 385-395.

Carmon, A., Nachson, I., Isseroff, A., \& Starinsky, R. (1976). Development aspects of visual hemifield differences in perception of verbal material. Brain \& Language, 3, 463-469.

Chastain, G. (1977). Feature analysis and the growth of a percept. Journal of Experimental Psychology: Human Perception \& Performance, 3, 291-298.

Coltheart, M., Curtis, B., Atkins, P., \& Haller, M. (1993). Models of reading aloud: Dual-route and parallel-distributed-processing approaches. Psychological Review, 100, 589-608.

Costello, A. D. L., \& Warrington, E. K. (1987). The dissociation of visuospatial neglect and neglect dyslexia. Journal of Neurology, Neurosurgery, \& Psychiatry, 50, 1110-1116.
Cubelli, R., Nichelli, P., Bonito, V., De Tanti, A., \& Inzaghi, M. G. (1991). Different patterns of dissociation in unilateral spatial neglect. Brain \& Cognition, 15, 139-159.

De Ruyter van Steveninck, R. R., Lewen, G. D., Strong, S. P., Koberle, R, \& Bialek, W. (1997). Reproducibility and variability in neural spike trains. Science, 275, 1805-1808.

Ellis, A. W., Young, A. W., \& Anderson, C. (1988). Modes of word recognition in the left and right cerebral hemispheres. Brain \& Language, 35, 254-273.

Estes, W. K., Allemeyer, D. H., \& Reder, S. M. (1976). Serial position functions for letter identification at brief and extended exposure durations. Perception \& Psychophysics, 19, 1-15.

Eviatar, Z. (1999). Cross-language tests of hemispheric strategies in reading nonwords. Neuropsychology, 13, 498-515.

FARID, M., \& Grainger, J. (1996). How initial fixation position influences visual word recognition: A comparison of French and Arabic. Brain \& Language, 53, 351-368.

Faust, M., Kravetz, S., \& BabKoff, H. (1993). Hemispheric specialization or reading habits: Evidence from lexical decision research with Hebrew words and sentences. Brain \& Language, 44, 254-263.

Fendrich, R, \& Gazzaniga, M. S. (1989). Evidence of foveal splitting in a commissurotomy patient. Neuropsychologia, 27, 273-281.

Forster, K. I., \& Chambers, S. M. (1973). Lexical access and naming time. Journal of Verbal Learning \& Verbal Behaviour, 12, 627-635.

Frederiksen, J. R, \& Kroll, J. F. (1976). Spelling and sound: Approaches to the internal lexicon. Journal of Experimental Psychology: Human Perception \& Performance, 2, 361-379.

Georgopoulos, A. P., Kalaska, J. F., Caminiti, R, \& Massey, J. T. (1982). On the relation between the direction of two-dimensional arm movements and cell discharge in the motor cortex. Journal of Neuroscience, 2, 1527-1537.

Grainger, J., \& JACOBS, A. M. (1991). Masked constituent letter priming in an alphabetic decision task. European Journal of Cognitive Psychology, 3, 413-434.

Green, D. W., \& Shallice, T. (1976). Direct visual access in reading for meaning. Memory \& Cognition, 4, 753-758.

Hammond, E. J., \& GREEN, D. W. (1982). Detecting targets in letter and non-letter arrays. Canadian Journal of Psychology, 36, 67-82.

Hellige, J. B. (1993). Hemispheric asymmetry: What's right and what's left? Cambridge, MA: Harvard University Press.

Hellige, J. B., \& Cowin, E. L. (1996). Effects of stimulus arrangement on hemispheric differences and interhemispheric interaction for processing letter trigrams. Neuropsychology, 10, 247-253.

Hellige, J. B., Cowin, E. L., \& ENG, T. L. (1995). Recognition of CVC syllables from LVF, RVF, and central locations: Hemispheric differences and interhemispheric interactions. Journal of Cognitive Neuroscience, 7, 258-266.

Hellige, J. B., \& Scott, G. B. (1997). Effects of output order on hemispheric asymmetry for processing letter trigrams. Brain \& Language, 59, 523-30.

Hellige, J. B., \& Yamauchi, M. (1999). Quantitative and qualitative hemispheric asymmetry for processing Japanese kana. Brain \& Cognition, 40, 453-463.

Hopfield, J. J. (1995). Pattern recognition computation using action potential timing for stimulus representation. Nature, 376, 33-36.

Humphreys, G. W., EvetT, L. J., \& Quinlan, P. T. (1990). Orthographic processing in visual word identification. Cognitive Psychology, 22, 517-560.

Joliot, M., Ribary, U., \& LlinAs, R. (1994). Human oscillatory brain activity near $40 \mathrm{~Hz}$ coexists with cognitive temporal binding. Proceedings of the National Academy of Sciences, 91, 11748-11751.

Katz, R. B., \& Sevush, S. (1989). Positional dyslexia. Brain \& Language, 37, 266-289.

Koriat, A. (1985). Lateralization effects in pointed and unpointed Hebrew. British Journal of Psychology, 76, 161-173.

Koriat, A., \& Norman, J. (1985). Reading rotated words. Journal of Experimental Psychology: Human Perception \& Performance, 11, 490-508.

Krumhansl, C. L., \& Thomas, E. A. C. (1976). Extracting identity and location information from briefly presented letter arrays. Perception \& Psychophysics, 20, 243-258.

Lefton, L. A., Fisher, D. F., \& Kunn, D. M. (1978). Left-to-right pro- 
cessing of alphabetic material is independent of retinal location. Bulletin of the Psychonomic Society, 12, 171-174.

Lisman, J. E., \& IDIART, M. A. P. (1995). Storage of $7 \pm 2$ short-term memories in oscillatory subcycles. Science, 267, 1512-1515.

Llinas, R, \& Ribary, U. (1993). Coherent 40-Hz oscillation characterizes dream state in humans. Proceedings of the National Academy of Sciences, 90, 2078-2081.

MarKs, N. L., \& Hellige, J. B. (1999). Effects of bilateral stimulation and stimulus redundancy interhemispheric interaction. Neuropsychology, 13, 475-487.

Mason, M. (1982). Recognition time for letters and nonletters: Effects of serial position, array size, and processing order. Journal of Experimental Psychology, 8, 724-738.

Mayzner, M. S., \& Tresselt, M. E. (1970). Visual information processing with sequential inputs: A general model for sequential blanking, displacement, and overprinting phenomena. In E. Harms \& M. E. Tresselt (Eds.), Third Conference on the Fundamentals of Psychology: Various approaches to the study of perception (Annals of the New York Academy of Sciences, Vol. 169, pp. 599-618). New York: New York Academy of Sciences.

McClelland, J. L., \& Rumelhart, D. E. (1981). An interactive activation model of context effects in letter perception: Part 1. An account of basic findings. Psychological Review, 88, 375-407.

Melamed, F., \& Zaidel, E. (1993). Language and task effects on lateralized word recognition. Brain \& Language, 45, 70-85.

Mewhort, D. J. K., \& BeAL, A. L. (1977). Mechanisms of word identification. Journal of Experimental Psychology, 3, 629-640.

Mewhort, D. J. K., Merikle, P. M., \& Bryden, M. P. (1969). On the transfer from iconic to short-term memory. Journal of Experimental Psychology, 81, 89-94.

Montant, M., Nazir, T. A., \& Poncet, M. (1998). Pure alexia and the viewing position effect in printed words. Cognitive Neuropsychology, 15, 93-140.

Mozer, M. C. (1987). Early parallel processing in reading: A connectionist approach. In M. Coltheart (Ed.), Attention and performance XII: The psychology of reading (pp. 83-104). London: Erlbaum.

Mozer, M. C., \& Behrmann, M. (1992). On the interaction of selective attention and lexical knowledge: A connectionist account of neglect dyslexia. Journal of Cognitive Neuroscience, 2, 96-123.

Orbach, J. (1967). Differential recognition of Hebrew and English words in right and left visual fields as a function of cerebral dominance and reading habits. Neuropsychologia, 5, 127-134.

O'Regan, J. K., Levy-Schoen, A., Pynte, J., \& Brugaillere, B. (1984). Convenient fixation location within isolated words of different length and structure. Journal of Experimental Psychology: Human Perception \& Performance, 10, 289-298.

Perea, M. (1998). Orthographic neighbors are not all equal: Evidence using an identification technique. Language \& Cognitive Processes, 13, 77-90.

Peressotti, F., \& Grainger,J. (1995). Letter-position coding in random consonant arrays. Perception \& Psychophysics, 57, 875-890.

Peressotti, F., \& Grainger, J. (1999). The role of letter identity and letter position in orthographic priming. Perception \& Psychophysics, 61, 691-706.

Plaut, D. C., \& Shallice, T. (1993). Deep dyslexia: A case study of connectionist neuropsychology. Cognitive Neuropsychology, 10, $377-500$.
Proctor, R. W., \& Healy, A. F. (1987). Task-specific serial position effects in comparisons of multiletter strings. Perception \& Psychophysics, 42, 180-194.

Reuter-Lorenz, P. A., \& BAynes, K. (1992). Modes of lexical access in the callosotomized brain. Journal of Cognitive Neuroscience, $\mathbf{4}$, $155-164$.

Rieke, F., Warland, D. [K.], De Ruyter van Steveninck, R., \& BIALEK, W. (1997). Spikes: Exploring the neural code. Cambridge, MA: MIT Press.

Seidenberg, M. S., \& McClelland, J. L. (1989). A distributed developmental model of word recognition and naming. Psychological Review, 96, 523-568.

Sieroff, E., Pollatsek, A. \& Posner, M. I. (1988). Recognition of visual letter strings following injury to the posterior visual spatial attentional system. Cognitive Neuropsychology, 5, 427-449.

Sieroff, E., \& Posner, M. I. (1988). Cueing spatial attention during processing of words and letter strings in normals. Cognitive Neuropsychology, 5, 451-472.

Strangert, B., \& BRÄNNSTRÖM, L. (1975). Spatial interaction effects in letter processing. Perception \& Psychophysics, 17, 268-272.

Sugishita, M., Hamilton, C. R, Sakuma, I., \& Hemmi, I. (1994). Hemispheric representation of the central retina of commissurotomized subjects. Neuropsychologia, 32, 399-415.

Sugishita, M., Hemmi, I., Sakuma, I., Beppu, H., \& Shiokawa, Y. (1993). The problem of macular sparing after unilateral occipital lesions. Journal of Neurology, 241, 1-9.

Titimen, H., Sinkmonen, J., Rainikainen, K., Alho, K., LaviKAINEN, J., \& NÄÄTÄNEN, R. (1993). Selective attention enhances the 40-Hz response in humans. Nature, 364, 59-60.

TownsEnd, J. T. (1971). A note on the identifiability of parallel and serial processes. Perception \& Psychophysics, 10, 161-163.

Trauzettel-Klosinski, S., \& Reinhard, J. (1998). The vertical field border in hemianopia and its significance for fixation and reading. Investigations in Ophthalmology \& Visual Science, 39, 2177 2186.

Victor, J. D., \& PUrpura, K. P. (1996). Nature and precision of temporal coding in visual cortex: A metric-space analysis. Journal of Neurophysiology, 76, 1310-1326.

WHITNEY, C. S. (2001). Position-specific effects within the SERIOL framework of letter-position coding. Manuscript submitted for publication.

Whitney, C. S. (in press). An explanation of the length effect for rotated words. Proceedings of the Fourth International Conference on Cognitive Modeling.

Whitney, C. S., \& Berndt, R. S. (1999). A new model of letter string encoding: Simulating right neglect dyslexia. Progress in Brain Research, 121, 143-163.

Whitney, C. S., Berndt, R. S., \& Reggia, J. A. (1996). Simulation of neurogenic reading disorders with a dual-route connectionist model. In J. A. Reggia, E. Ruppin, \& R. S. Berndt (Eds.), Neural modeling of brain and cognitive disorders (pp. 201-228). Singapore: World Scientific.

Wolford, G., \& Hollingsworth, S. (1974). Retinal location and string position as important variables in visual information processing. Perception \& Psychophysics, 16, 437-442.

Young, A. W., \& Ellis, A. W. (1985). Different modes of lexical access for words presented in the left and right visual hemispheres. Brain \& Language, 24, 326-358. 


\section{APPENDIX A \\ Locational Gradient}

We denote the activation of a feature node as $F$. For simplicity, we consider all feature nodes comprising a single letter to reach a similar level of activation, which is determined by the letter's string position, $P$, and retinal location of its center, $R(P)$. For notational convenience, we will write $R$ rather than $R(P)$ in the following. We first consider feature activations in the absence of hemispheric interaction, denoted $F_{h}$. For simplicity, we assign fixation, $R=0$, to a single hemisphere-namely, the RVF/LH. $F_{h}$ is determined by the combination of bottom-up excitatory input, $E$, and lateral inhibitory input, $I$, and is restricted to a maximal value, denoted by constant $c_{M}$. That is,

$$
F_{h}(P, R)=M(E(R)-I(P, R)),
$$

where $M(x)=\min \left(c_{M}, x\right)$.

Bottom-up excitatory input is a function of acuity, denoted $C$, and visual field:

$$
E(R)= \begin{cases}C(R) & \text { if } R \geq 0 \\ c_{E} * C(R) & \text { if } R<0,\end{cases}
$$

where $c_{E}$ is a constant $\gg 1$, reflecting our assumption of stronger excitatory input to the LVF/RH.

Lateral inhibitory input is the sum of inhibitory inputs from features having preferred locations to the left of $R$. This quantity increases with the number of such features, their activation levels, and the strengths of the inhibitory connections. Rather than directly modeling the feedback processes underlying such lateral inhibition, we approximate the amount of inhibitory input as the activation of the leftmost letter's features weighted by an increasing function of the number of letters to the left of $R$.

The leftmost letter designates the letter that lies farthest to the left within the same visual field as $R$. Its position, denoted $P_{l}$, is 1 if the first letter of the string lies in the same visual field as $R$; otherwise, it is the position of the letter occurring at fixation, because then $R$ is in the RVF, and $R(1)$ is not. Thus, we have

$$
I(P, R)=M\left(E\left(R\left(P_{l}\right)\right)\right) * W\left(P-P_{l}, \mathrm{R}\right)
$$

where $M\left(E\left(R\left(P_{l}\right)\right)\right)$ gives the feature activation of the leftmost letter (which does not receive any inhibition), and $W$ denotes the weighting function. $W$ increases as the number of letters to the left (given by $P-P_{l}$ ) increases. $W$ also depends on hemisphere; inhibitory connections are stronger in the LFV/RH than in the $\mathrm{RVF} / \mathrm{LH}$ (as is necessary to invert the acuity gradient). That is, for a given $P-P_{l}, W$ is larger for $R<0$ than for $R>0$.

The individual hemispheric gradients are joined via inhibition of the RVF/LH's feature nodes by an amount proportional to the number of letters coming from the LVF/RH. That is,

$$
F(P, R)= \begin{cases}F_{h}(P, R) & \text { if } R<0 \text { or } R(1) \geq 0 \\ F_{h}(P, R)-c_{F} *\left(P_{l}-1\right) & \text { otherwise, }\end{cases}
$$

Table A1

Definition of $W$

\begin{tabular}{ccc}
\hline$P-P_{l}$ & $R \geq 0$ & $R \leq 0$ \\
\hline 0 & 0.00 & 0.00 \\
1 & 0.15 & 0.80 \\
2 & 0.25 & 1.10 \\
3 & 0.30 & 1.25 \\
4 & 0.50 & 1.35 \\
5 & 0.50 & 1.45 \\
6 & 0.50 & 1.65 \\
\hline
\end{tabular}

where $c_{F}$ is a positive constant. This yields a decreasing gradient, such that $F(P, R)>F(P+1, R(P+1))$.

The modeled results discussed in Section 3.1 were carried out as follows. To instantiate this model, values for the constants $c_{M}, c_{E}$, and $c_{F}$ and definitions of the functions $C$ and $W$ must be supplied. The displayed results used the following definitions, where $R$ is in units of letter width:

$$
\begin{aligned}
& c_{M}=1.0 \quad c_{E}=1.8 \quad c_{F}=0.2 \\
& C(0)=1.1 \\
& C(|R|)=C(|R|-1)-C_{\text {diff }}(|R|) \\
& C_{\text {diff }}(|R|)= \begin{cases}0.1 & \text { if } 0<|R| \leq 3 \\
0.07 & \text { if } 3<|R| \leq 6 \\
0.05 & \text { if } 6<|R| \leq 9 \\
0.04 & \text { if } 9<|R| .\end{cases}
\end{aligned}
$$

The definition of $W$ is best displayed in tabular form (see Table A1).

These definitions specify the feature level activations for a given retinal location and string position. We assume that the letter level activations (positional gradient) have a similar shape to the locational gradient (except at the final letter, which is not being considered here). We converted the feature activations to letter activations, $L$, and then to perceptibility, $P_{L}$, in the following way:

$$
\begin{aligned}
& L(P, R)= \begin{cases}F(P, R)+0.2 & \text { if } P=1 \\
F(P, R) & \text { if } P>1\end{cases} \\
& P L(P, R)=\min (100, \max (0,100 * L(P, R))) .
\end{aligned}
$$

For simplicity, we set letter level activations to be equivalent to feature level activations, except at the first position (in order to give a better fit to the data, which does not affect the overall shape of the curves). Multiplying letter activation by 100 and bounding it between 0 and 100 gives the modeled value, $P L$, for percentage of correct recognitions, as displayed in Figure 4.

Next, we consider error patterns for horizontal presentation of trigrams, as discussed in Section 3.3. The trigrams were presented such that their middle letters were the same distance from fixation in each visual field. We denote the retinal location of the first letter in the RVF as $R_{N}$ (near fixation) and the retinal location of the third letter in the RVF as $R_{F}$ (far from fixation). In the LVF, the first letter then has location $-R_{F}$, and the third letter has location $-R_{N}$.

We assume the probability of correct recognition of a letter is proportional to its feature level activation. We assume this is true even for the final letter, because presentation durations were short enough ( $\approx 50 \mathrm{msec})$ to push the firing of the final letter near the end of the oscillatory cycle, removing a final-letter advantage. We are interested in the proportion of initial-letter recognitions versus final-letter recognitions, across visual fields. We calculate an asymmetry measure, $A$, by

$$
\begin{aligned}
A\left(R_{N}, R_{F}\right)= & \left(F_{h}\left(1,-R_{F}\right)-F_{h}\left(3,-R_{N}\right)\right) \\
& -\left(F_{h}\left(1, R_{N}\right)-F_{h}\left(3, R_{F}\right)\right) .
\end{aligned}
$$

That is, for each visual field we subtract the number of finalletter recognitions from the number of initial-letterrecognitions, to get a recognition slope. The recognition slope of the RVF is compared with that of the LVF by subtracting it from that of the LVF. If $A$ is 0 , there is no asymmetry. $A$ increases as the propor- 


\section{APPENDIX A (Continued)}

tion of final-letter recognitions in the LVF decreases relative to those in the RVF - that is, as the visual field asymmetry increases.

Next, we derive expressions for the $F_{h}$ s from the definitions above. We assume that the stimuli are far enough from fixation that the bounding by function $M$ is not necessary. We get

$$
\begin{aligned}
& F_{h}\left(1,-R_{F}\right)=c_{E} * C\left(R_{F}\right) \\
& F_{h}\left(3,-R_{N}\right)=c_{E} * C\left(R_{N}\right)-W\left(2,-R_{N}\right) * c_{E} * C(R F) \\
& F_{h}\left(1, R_{N}\right)=C\left(R_{N}\right) \\
& F_{h}\left(3, R_{F}\right)=C\left(R_{F}\right)-W\left(2, R_{F}\right) * C\left(R_{N}\right) .
\end{aligned}
$$

Substituting into the definition of $A$ and combining terms yields

$$
\begin{aligned}
A\left(R_{N}, R_{F}\right)= & \left(c_{E}+W\left(2,-R_{N}\right)+1\right) * C\left(R_{F}\right) \\
& -\left(c_{E}+W\left(2, R_{F}\right)+1\right) * C\left(R_{N}\right) .
\end{aligned}
$$

We are interested in the value of $A$ for the stimulus location used by Hellige and Cowin (1996) versus that used by Eviatar (1999). In the former experiment, the far edge of the stimulus fell $3.0^{\circ}$ of visual angle from fixation, and the near edge fell $1.5^{\circ}$ from fixation. In the latter experiment, the locations were $3.0^{\circ}$ and $1.0^{\circ}$, respectively. The difference in the locations of the far letters' centers (resulting from letter size) is negligible; we consider the $R_{F}$ s to be the same across experiments. This yields the same value for the first term of $A$ for both experiments. Only the sign of the second argument to $W$ matters, so the fact that the $R_{N}$ s differed does not affect the value of $W\left(2,-R_{N}\right)$. Superscripting to denote experiment, $R_{N}^{\mathrm{H}}>R_{N}^{\mathrm{E}}$, so $C\left(R_{N}^{\mathrm{H}}\right)<C\left(R_{N}^{\mathrm{E}}\right)$. Thus, the second term is smaller for $A^{\mathrm{H}}$ than for $A^{\mathrm{E}}$, so $A^{\mathrm{H}}>A^{\mathrm{E}}$. This analysis is consistent with the results; the Hellige and Cowin experiment showed asymmetry, whereas the Eviatar experiment did not.

\section{APPENDIX B}

\section{Model of Brysbaert (1994)}

We model the naming latency, denoted $R T$, as the sum of the times required for transfer, inversion, phonological assembly, and all other processing. That is,

$$
\begin{aligned}
R T(h, \text { len }, \text { fix })= & T_{t r}(T R(h, f i x))+T_{i n}(T R(h, f i x), I N V(f i x)) \\
& +c_{P} * \text { len }+c_{B},
\end{aligned}
$$

where $R T$ is a function of the dominant hemisphere $(h)$, word length (len), and fixation point (fix). $T_{t r}$ is the time cost of interhemispheric transfer, which is a function of the number of letters to transfer, $T R . T_{\text {in }}$ is the time cost of acuity gradient inversion, which is a function of $T R$ and the number of letters to invert, $I N V$. The quantity $c_{P} *$ len corresponds to phonological assembly time, where $c_{P}$ is a positive constant. The constant $c_{B}$ denotes the base time required for all other processing.

The number of letters to transfer, $T R$, depends on both fixation point and dominant hemisphere; when the word appears in the visual field projecting to the dominant hemisphere, $T R=0$; otherwise, $T R=$ len. As explained in Section 3.2, it is assumed that transfer time does not vary with the number letters to be transferred; that is, $T_{t r}(x)=c_{T}$ for $x>0$, where $c_{T}$ is a positive constant, while $T_{t r}(0)=0$. Thus,

$$
T R(h, \text { fix })=\left\{\begin{array}{ll}
0 & \text { if } h=\mathrm{LH} \text { and } f i x=1 \\
0 & \text { if } h=\mathrm{RH} \text { and } f i x=l e n \\
l e n & \text { if } h=\mathrm{LH} \text { and } \text { fix }=l e n \\
l e n & \text { if } h=\mathrm{RH} \text { and } \text { fix }=1
\end{array} .\right.
$$

and

$$
T_{t r}(T R)= \begin{cases}0 & \text { if } T R=0 \\ c_{T} & \text { if } T R=\text { len }\end{cases}
$$

The number of letters to invert, $I N V$, depends only on the fixation point; for initial-letter fixation, $I N V=0$ (because the word falls into the RVF/LH); for final-letter fixation, INV = len (because the word falls into the LVF/RH). That is,

$$
I N V(f i x)= \begin{cases}0 & \text { if } f i x=1 \\ l e n & \text { if } f i x=l e n\end{cases}
$$

It is assumed that $T_{i n}$ increases with increasing $I N V$ (i.e., the time required for acuity gradient inversion increases with the number of letters to invert, as proposed in Section 3.2). We also assume that $T_{i n}(0, I N V)<T_{i n}(l e n, I N V)$ (i.e., the time cost of inversion increases if transfer is also necessary). This assumption indicates that the cost of both inversion and transfer is greater than the sum of inversion only and transfer only. Such an assumption is necessary to fit the data; moreover, it seems reasonable to assume that nonlinear effects arise when feature information is degraded by both inversion and transfer.

The displayed results for $R T$ in Figure 5 instantiated the model using the following definitions of the constants $c_{T}$ (hemispheric transfer time), $c_{P}$ (phonologicalassembly time per letter), and $c_{B}$ (base processing time), and the function $T_{\text {in }}$ (inversion time):

$$
\begin{aligned}
& c_{T}=9 \quad c_{P}=3 \quad c_{B}=429 \\
& T_{i n}(T R, I N V)= \begin{cases}0 & \text { if } I N V \leq 3 \\
T_{i n}(I N V) & \text { if } I N V>3 \text { and } T R=0, \\
1.7 * T_{\text {in }}(I N V) & \text { if } I N V>3 \text { and } T R>0\end{cases}
\end{aligned}
$$

where

$$
T_{i n}(I N V)=(I N V-3)^{2}+4 .
$$

The modeled results are consistent with the experimentaldata. For final-letterfixation in LH-dominant readers, reaction times increase steeply with increasing length due to the derivative of the function $T_{\text {in }}$ (len, len). For RH-dominant readers, the cost of finalletter fixation is not as steep because $T_{i n}(0$, len $)<T_{\text {in }}($ len,len $)$. In fact, for three-letter words, a trend toward an LVF/RH advantage for RH-dominant readers appears because $T_{i n}(0,3)<$ $T_{t r}(3)$. However, as len increases, an RVF/LH advantage emerges, as $T_{\text {in }}(0$, len $)$ increases while $T_{t r}(0$, len $)$ stays constant. 